\title{
Contents
}

17. Aerial Collective Systems 1

Jean-Christophe Zufferey, Sabine Hauert, Timothy Stirling,

Severin Leven, James Roberts, Dario Floreano

17.1 Introduction . . . . . . . . . . . . . . . . . . . . 1

17.2 Flying Robots . . . . . . . . . . . . . . . . . 2

17.2.1 Platforms . . . . . . . . . . . . . . . 2

17.2.2 Flight Stabilisation . . . . . . . . . . . . . . . 6

17.2.3 Localisation and Navigation . . . . . . . . . . . . . 9

17.2.4 Collision Avoidance . . . . . . . . . . . . . . . . 12

17.2.5 Power Management and Energy . . . . . . . . . . . . . . . 13

17.3 Enabling Coordination . . . . . . . . . . . . . . . 16

17.3.1 Communication . . . . . . . . . . . . . 16

17.3.2 Relative Positioning . . . . . . . . . . . . . . . 19

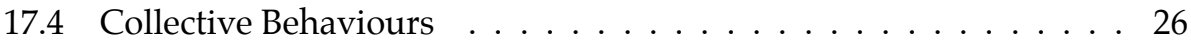

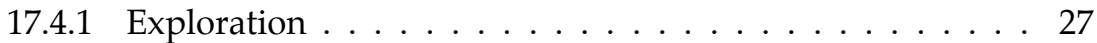

17.4.2 Flocking . . . . . . . . . . . . . . 31

17.4.3 Approaches to Controller Design . . . . . . . . . . . 34

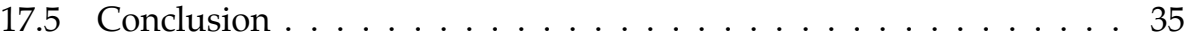




\section{Aerial Collective Systems}

Jean-Christophe Zufferey, Sabine Hauert, Timothy Stirling,

Severin Leven, James Roberts, Dario Floreano

\subsection{Introduction}

Deployment of multiple flying robots has attracted the interest of several research groups in recent times both because such a feat represents many interesting scientific challenges and because aerial collective systems have a huge potential in terms of applications.

By working together, multiple flying robots can perform a given task quicker or more efficiently than a single system (Sahin, 2005; Beni, 2005). Furthermore, multiple robots can share computing, sensing and communication payloads thus leading to lighter robots that could be safer than a larger system, easier to transport and even disposable in some cases (Holland et al., 2005). Deploying a fleet of unmanned aerial vehicles instead of a single aircraft allows rapid coverage of a relatively larger area or volume (Alidaee et al., 2009; Beard et al., 2006; Bertuccelli et al., 2004; Gancet et al., 2005; Kovacina et al., 2002; Kuiper and Nadjm-Tehrani, 2006; Lawrence et al., 2004; Merino et al., 2006; Parunak et al., 2005; Richards et al., 2002; Sauter et al., 2005).

Collaborating airborne agents can help each other by relaying communication or by providing navigation means to their neighbours (Hauert et al., 2008, 2009a; Stirling et al., 2010). Flying in formation provides an effective way of de-congesting the airspace. Aerial swarms also have an enormous artistic potential because they allow creating physical 3D structures that can dynamically change their shape over time (Nembrini et al., 2005; Oung et al., 2010).

However, the challenges to actually build and control aerial swarms are numerous. First of all, a flying platform is often more complicated to engineer than a terrestrial robot because of the inherent weight constraints and the absence of 
a mechanical link with any inertial frame that could provide mechanical stability and state reference. In the first section of this chapter, we therefore review these challenges and provide pointers to state-of-the-art methods to solve them. Then as soon as flying robots need to interact with each other, all sorts of problems arise such as wireless communication from and to rapidly moving objects and relative positioning. The aim of Sec. 17.3 is therefore to review possible approaches to technically enable coordination among flying systems. Finally, Sec. 17.4 tackles the challenge of designing individual controllers that enable a coherent behaviour at the level of the swarm. This challenge is made even more difficult with flying robots because of their 3D nature and their motion constraints that are often related to the specific architectures of the underlying physical platforms. This section is complementary to the rest of this book since it focusses only on methods that have been designed for aerial collective systems.

\subsection{Flying Robots}

Flying robots are particular in that they have to satisfy the most stringent constraints in terms of weight and integration. They have to carry all the necessary sensor and computing power while still being able to lift themselves for a reasonable amount of time. Depending on the application, the flying platforms will look very different if they need to fly in closed settings or high in the sky, if they need to physically interact with the environment, if they must stay airborne for hours or just fly for small periods of time. This section provides a survey of possible platform architectures together with their advantages and limitations in the perspective of collective robotic applications. There is one aspect that is shared among most flying platforms: since flying systems are by nature not in contact with any inertial frame, they need to actively control their orientation and position using sensory feedback at relatively high frequency. The second part of this section therefore provides an overview of flight stabilisation techniques before surveying possible approaches to localisation and navigation. Discussed subsequently is the issue of collision avoidance, which is of crucial importance for flying systems. To conclude this section an overview of power management and techniques for mitigating the severely limited energy autonomy of flying robots is presented. For complementary information or for a review of relatively larger unmanned aerial vehicles and related control techniques, the reader is referred to the book chapter on aerial robotics by Feron and Johnson (2008).

\subsubsection{Platforms}

Constructing lighter-than-air structures is probably the easiest way of getting a system airborne since there are no critical aerodynamic issues. In the history of human flight, the hot-air balloon invented by the Montgolfier brothers was the first to successfully take-off already in 1783, well before the advent of aeroplanes and helicopters. Airships are not only good for their simplicity, they are also notably economical when it comes to lifting heavy loads. The main limitation of airships is 
linked to their huge lateral surface that will inevitably produce drag forces as soon as the airspeed departs from zero. This is why airships are usually very sensitive to wind and cannot travel efficiently at high speeds. However, because of their ability to naturally float in the air, small airships (also called blimps when there is no rigid structure inside the hull) have been widely used as research platforms in aerial robotics (Bermudez i Badia et al., 2005; Iida, 2003; Van Der Zwaan et al., 2002; Zhang and Ostrowski, 1998; Zufferey et al., 2006a). In particular, Melhuish and Welsby (2002) have been using small indoor blimps to demonstrate flocking behaviours (Fig. 17.1.) and Nembrini et al. (2005) have been designing cubical airships named "Mascarillons" or "SAILS" for artistic shows (Fig. 17.2.) with the goal of enabling collective behaviours.

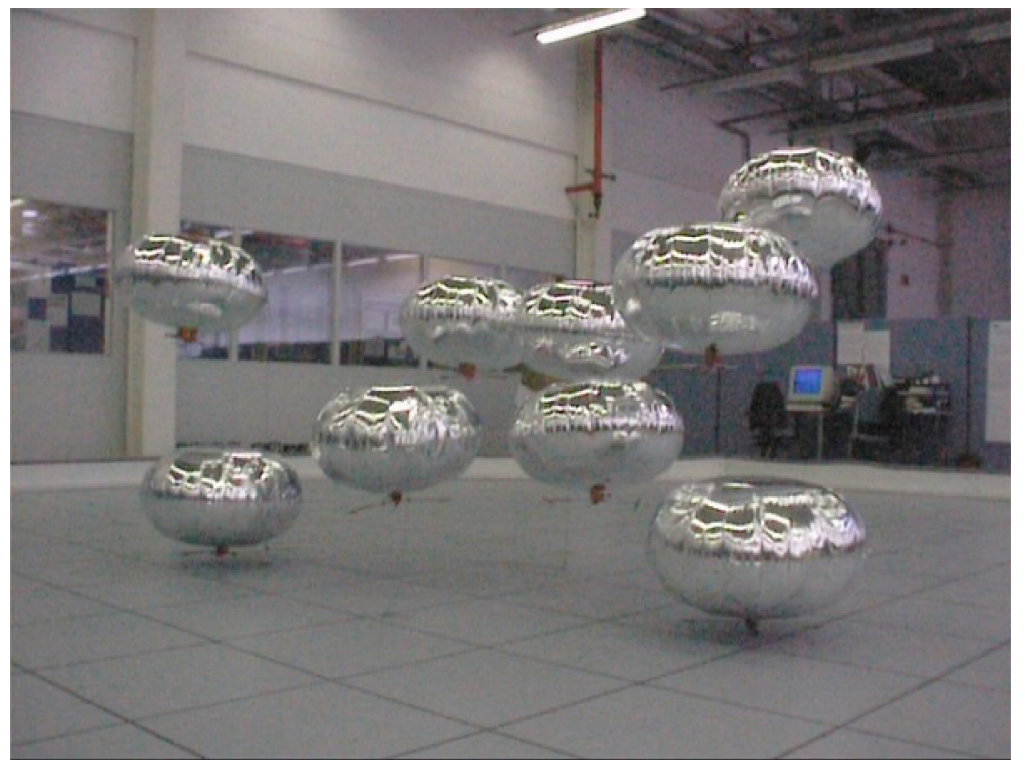

Figure 17.1. The swarm of 9 miniature blimps used to study flocking behaviour in an indoor settings using embedded infrared relative positioning sensors. Image courtesy of Chris Melhuish, Bristol Robotics Laboratory, University of Bristol and the West of England, Bristol.

Fixed-wing aeroplane architectures are widely spread in robotics thanks to their simple mechanical design and energetic efficiency when it comes to travelling relatively long distances (Tennekes, 1997). Most military unmanned aerial vehicles (UAV) fall into this category and several research projects involving a fleet of fixed-wing UAVs have been successfully carried out (Allred et al., 2007; Beard et al., 2006; Cole et al., 2006; How et al., 2004). The use of no-tail or flying-wing geometries (Fig. 17.3. and Fig. 17.4.) has recently gained a lot of interest in the domain thanks to its mechanical simplicity. However, fixed-wing aeroplane dynamics are known to be nonholonomic because their trajectory is mostly defined by the orientation of their main axis. In normal flight regimes, the turn-rate of fixed-wing aeroplanes is indeed imposed by the inclination around their main axis (i.e. the roll axis) and cannot be changed instantaneously. In addition and contrarily to terrestrial robots, aeroplanes cannot slow down below a certain velocity known as 


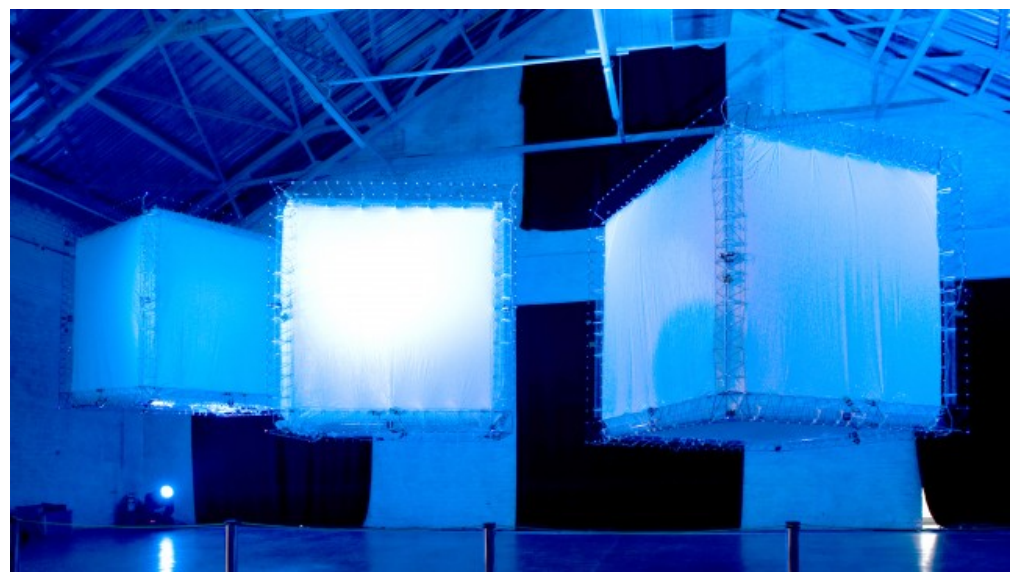

Figure 17.2. Three aerobots of the VOILES SAILS project, model m225c "Tryphon". Image courtesy Nicolas Reeves and David St-Onge, NXI Gestatio Design Lab, HexagramCIAM Institute, University of Quebec, Montreal. Photo: Asya Ablogina.

the stall speed. They are therefore incapable of hovering or moving backwards. These strong motion constraints do not facilitate the implementation of collective behaviours and must be carefully taken into account when designing cooperative controllers (Beard et al., 2006; Qu, 2009).

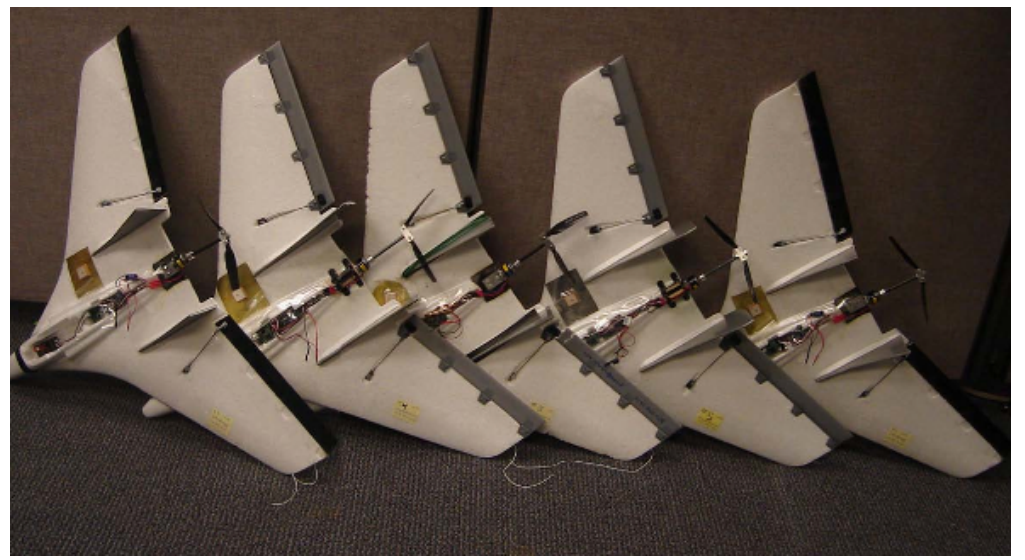

Figure 17.3. The University of Colorado custom-developed flying wings. The design is relatively simple, so scaling to produce larger flocks can be quickly achieved. Image courtesy of William Pisano and Dale Lawrence, Orbital Systems Lab, University of Colorado, Boulder.

Helicopters are of interest in the field of collective robotics since they can hover, as well as fly backwards and sideways, which facilitates the design of cooperative controllers $(\mathrm{Qu}, 2009)$. However, the mechanical complexity of standard architecture including a main rotor with a swash plate and tail rotor is relatively costly to produce and maintain. This is why most robotic projects have adopted multi-rotor configurations (Bouabdallah and Siegwart, 2007; Ducard and D'Andrea, 2009; Hoffmann et al., 2004), which rely on differential variations of thrust among the 


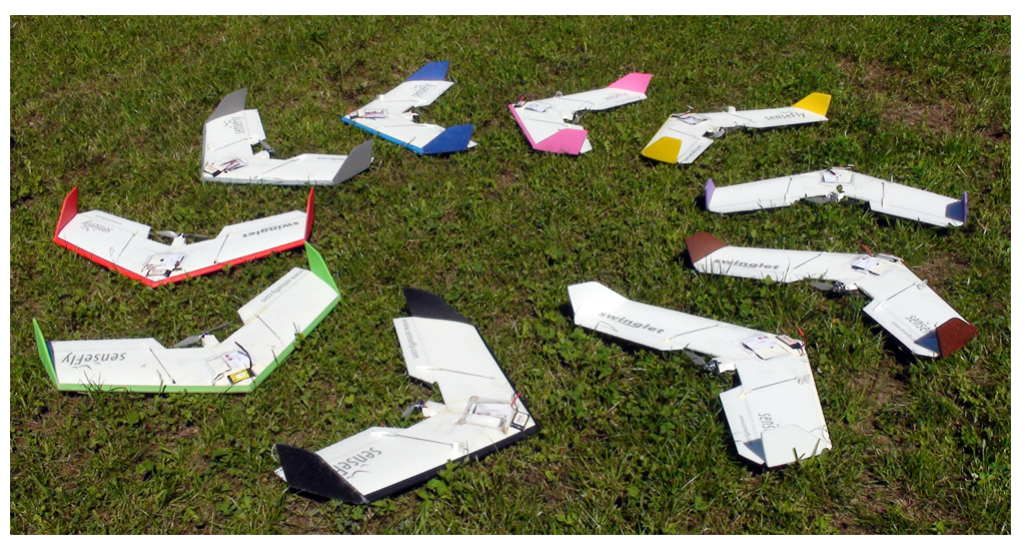

Figure 17.4. 10 swinglets produced in collaboration with senseFly LLC for the Swarming MAVs project at EPFL (see Sec. 17.4 for further details). These $80 \mathrm{~cm}$ aeroplanes are sufficiently lightweight (500 grams) so that they can be deployed over populated areas without much risks for people on the ground.

rotors in order to control all axes or rotation. Such configurations allow to get rid of the swash plate and feature impressive manoeuvrability at a cost of a slightly higher energy consumption. Quite a few projects have been using quadrotors (Fig. 17.5.) for collective operation (Hoffmann et al., 2004; Roberts et al., 2008; How et al., 2008). The downside of helicopters with respect to aeroplanes is that they require much more power to remain airborne, since the high rotation speed of the blades decreases their efficiency as air-foils (Gordon, 2006). As an example, electrically powered helicopters of less than a kilogram can typically remain airborne for 15 to 30 minutes only, whereas aeroplanes of comparable weight can stay in the air for at least twice this amount of time. One way of working around this limitation is to exploit the fact that helicopters do not need well-prepared runways to take-off and land in order to have them rest from time to time while they are carrying out their mission (Stirling et al., 2010). This can be done either by landing on the ground, or attaching to some objects such as ceilings of indoor environments (Roberts et al., 2008).

Flying platforms cannot always be categorised into airships, aeroplanes or helicopters. Some alternative designs have been proposed in the history of aviation and roboticists tend to be even more imaginative since they are not confronted with the same security rules as in commercial aviation. Covering all possible designs is beyond the scope of this chapter. However, it is worth citing a few noticeable trends in aerial robotic platform design. For instance, there is an increasing number of researchers looking at bio-inspired airframes with flapping wings to produce both lift and forward thrust (see for review Floreano et al. (2009)). Such architectures may lead to an interesting compromise as they enable both hovering and gliding flight. However, the energy efficiency of such solutions is still not comparable to that of rotorcraft (Lentink, 2008; Lentink et al., 2009). Other researchers have looked at flexible wings to better withstand wind gusts (Lawrence et al., 2004) or retractable wings to be able to efficiently penetrate cluttered environments while moving on the ground (Bachmann et al., 2005). 


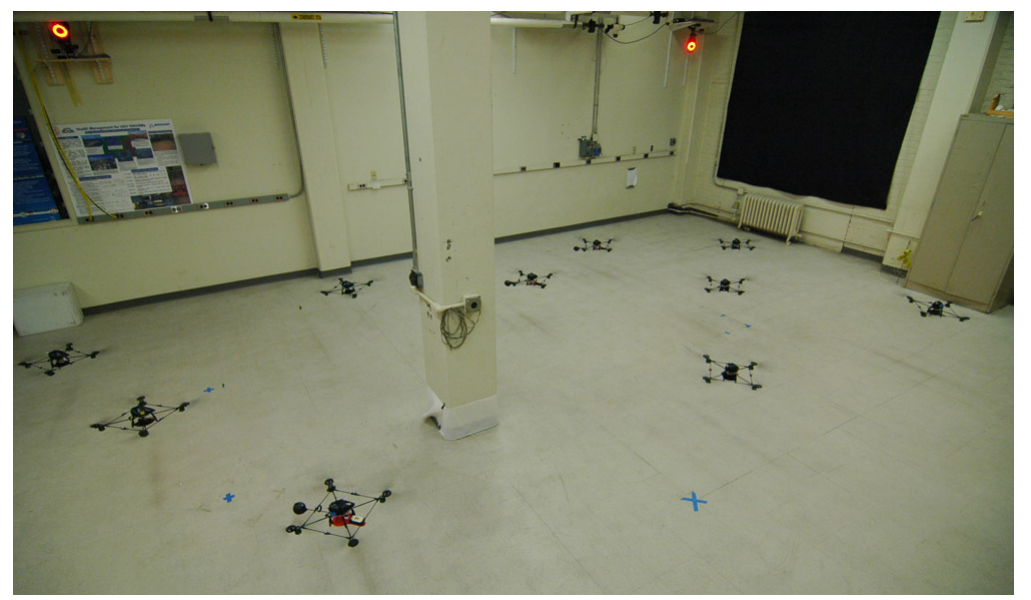

Figure 17.5. Eight autonomous quadrotors relying on an external positioning system for accurate flight control (see Sec. 17.2.3 for more details). Image courtesy of Jonathan How, Aerospace Controls Laboratory, Massachusetts Institute of Technology, Cambridge.

Other research avenues in flying platform design look at how robots can perch or attach to elevated objects (Cory and Tedrake, 2008; Kovac et al., 2010; Wickenheiser and Garcia, 2008) or even withstand crashes (Klaptocz et al., 2010) by adding intelligently designed exoskeletons. However, the authors are not aware of any collective operation of such alternative flying platforms at this point.

\subsubsection{Flight Stabilisation}

Flying robots are complex dynamic systems that operate in the 3D-space. Their motion is characterised by 6 degrees of freedom (DoF) for position and attitude, the relationship between system inputs, internal states and outputs is often nonlinear and the dynamics for single axes may strongly interact. Additional complexity derives from environmental challenges: wind gusts, thermals etc. Flight control of such systems is typically organised in layers, where the most basic layer is concerned with flight stabilisation (also called Stability Augmentation System (SAS)), meaning stabilisation of rotational and linear motion. Depending on the type of flying vehicle and its intrinsic stability, more or less effort for artificial stabilisation is required. While classical fixed-wing type vehicles may not need any artificial flight stabilisation at all, helicopter type vehicles instead will typically demand continuous attitude stabilization.

Classical aircraft control techniques (Etkin and Reid, 1995; Pratt, 2000; Stevens and Lewis, 2003) typically rely on state estimation data provided by an attitude and heading reference system (AHRS). Available as commercial products in various shapes, AHRSs involve a multitude of sensors and complex computation. Typical form factors range down to match-box size and below, with weights smaller than 15g. Complementary or Bayesian filters such as a Kalman filter, e.g. in Marins et al. (2001), are used to fuse data from orthogonally mounted rate gyros, accelerometers and magnetic sensors. Typical magnitudes of the attitude estima- 
tion error are $5-10^{\circ}$. For airspeed and altitude, additional sensors have to provide appropriate measurements (usually pressure transducers or GPS). Considering the restricted computational power of small-scale embedded processors, simplifications of the attitude estimation process have for example been proposed by Euston et al. (2008).

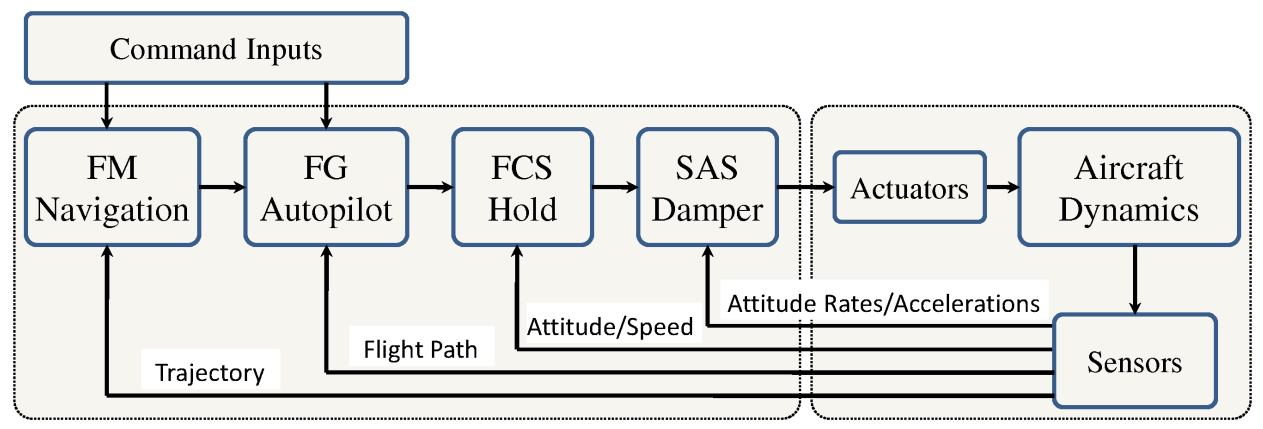

Figure 17.6. Classical flight control structure with nested control loops. The innermost loop is the Stability Augmentation System (SAS), mainly responsible for damping pitch, roll and yaw motion. In the second loop, the Flight Control System (FCS) provides hold functions for attitude angles and speed. Flight Guidance (FG) contains autopilot (AP) modes like altitude, turn control and auto-throttle (ATHR). Finally, Flight management (FM) in the fourth loop handles trajectory tracking. Except for rare situations (e.g. emergencies), input from a pilot or operator typically only affects the FM and FG control loops. Adapted from Fielding and Luckner (2000).

The most popular implementations of control architectures use AHRS sensor data as feedback to several nested control loops, as depicted in Fig. 17.6.. Controllers are typically implemented as PID controllers (Beard et al., 2005; Jang and Tomlin, 2001; Kim et al., 2006; Kingston and Beard, 2004). Other researchers have looked at control architectures based on fuzzy-logic control (Wu et al., 2005) or neural nets (Oh et al., 2004).

In order to reduce the complexity and cost of approaches using full state estimation by AHRSs, alternative approaches have been investigated as well. These are especially interesting for collective systems, where the cost of each individual vehicle becomes a prohibitive factor. A 3-sensor autopilot and control system is presented by Pisano and Lawrence (2007) that cannot only do flight stabilisation but also simple navigation based on a GPS. The use of low-cost infrared sensors for attitude estimation is described by Brisset et al. (2006) in the frame of the "Paparazzi" project, a very popular and simple open-source autopilot implementation. A minimalist control solution has been developed by Leven et al. (2009), which provides an easy-to-use interface to the three basic functionalities of airspeed, altitude and turn-rate control for a fixed-wing platform while only relying on a minimum of sensors and reactive control loops. Reactive flight stabilisation has further been achieved with bio-inspired techniques like optic flow: attitude, altitude and turn control using the optic flow measured by several small on-board cameras have been shown for indoor (Zufferey et al., 2006b) and outdoor (Beyeler et al., 2009; Zufferey et al., 2010) operation. While stabilisation solutions based on inertial sensors (rate gyros, accelerometers) work in every environment, optic flow 
techniques can only be used in contrast-rich environments, e.g close to the ground.

Flight stabilisation on rotary wing aircraft generally requires different approaches than on fixed wing aircraft. Stabilisation can be done either mechanically or electronically and usually there is a trade-off between stability and controllability.

Mechanically stabilised systems utilise the natural gyroscopic and/or aerodynamic effects created by a rotating mass or air-foil section respectively (Hirosue et al., 2003). This means that they require minimal sensors for stabilisation. The most common example of this is the contra-rotating helicopter with passive stabiliser bar (Holland et al., 2005; Matsue et al., 2005; Ohkura et al., 2005; De Nardi et al., 2006), which can be found in almost every hobby/toy shop around the globe. The top rotor is coupled to a weighted fly-bar and hinged in a way that controls the angle of attack of the rotor blades, the gyroscopic forces of this bar and the gravitational forces acting on it allow it to act as both a 2-axis gyroscope, for pitch and roll, and 3D accelerometer. Therefore, for complete stabilisation only a gyroscopic sensor for yaw rate is required. The problem with this kind of stabilisation is that the forces acting to keep the system stable also act against any control input that is given, thus limiting the amount of controllability, which also raises an issue with external disturbances such as wind. If the hinge on the rotor saturates then stability is lost and the aircraft may find itself in an unstable state.

For systems not using mechanical stabilisation, electronic stability controllers are required. A real-time electronic controller stabilises the platform by closing the loop between the actuation system and the attitude sensing. The bandwidth of the platforms dynamics, $\mathrm{fd}(\mathrm{Hz})$, is related to the speed at which the feedback system should track the sensor input and be able stabilise the platform. Generally, as a rule of thumb, the controllers sampling period, $\tau$, should be between (Eqn.17.1, Levine (1996)) :

$$
\frac{1}{30 \cdot f d}<\tau<\frac{1}{5 \cdot f d}
$$

Most gyroscopes, required for sensing the critical rotational dynamics of a platform, have a bandwidth limited to approximately $50 \mathrm{~Hz}$. If we select the minimum recommended sampling rate of 5 times (17.1) we obtain a minimum controller sampling frequency of $250 \mathrm{~Hz}$. To utilise the full bandwidth of the sensor, the sampling frequency can be made equal to the control step frequency. The problem here is finding an actuator that is capable of such speeds.

Many rotary wing aircraft such as classical helicopters, ducted-fans (Oh et al., 2005), hovering fixed wings (Green and Oh, 2005, 2006, 2008) etc. run into this problem because they are using standard $\mathrm{R} / \mathrm{C}$ servos for actuation. Unfortunately, these servos have an upper bandwidth limitation of approximately $50 \mathrm{~Hz}$, which is defined by the pulse width modulated signal used to set its position. On such systems, a more complex controller, typically non-linear in design, may be necessary. These types of controllers generally require an accurate model of the system's dynamics to perform well. 
On the other hand, if we solve the problem of actuator bandwidth, it is possible to increase the speed of the control system to obtain more precisely the bandwidth response of the sensor, reduce the complexity of the controller and eliminate the need for a dynamic model.

With the emergence of high speed brushless motor electronic speed controllers, capable of $500 \mathrm{~Hz}$ or greater (Gurdan et al., 2007), this is now possible. This has lead to the emergence of multi-rotor aircraft, like quadrotors (Bouabdallah et al., 2005; Gurdan et al., 2007; Hoffmann et al., 2004, 2007; Waslander et al., 2005), hexarotors and octorotors (Romero et al., 2007; Salazar et al., 2009), using simple controllers, such as a Proportional-Derivative, which have recently become very popular as simple control and hardware can be appealing.

Several other aspects of flight stabilisation have been studied. While the adjustment of controller parameters is often done manually, some researchers have investigated automatic parameter adjustment (optimal control) and controller robustness to sensor / actuator failures (Shore and Bodson, 2004) or sudden changes in the aerodynamics (Knoebel et al., 2006). Also, techniques from adaptive control theory have been employed to design controllers for a broad flight envelope instead of a single operating point, like for example sliding or switching mode PID controllers (Andrievsky and Fradkov, 2003).

Given the many different flavours of existing flight stabilisation techniques, the particular choice is often based on personal preferences. For collective aerial systems, though, it is certainly advantageous to implement low-cost and simple systems that have small maintenance requirements and are easy to use in terms of configuration and flight operation. Aerial vehicles have also strong size and weight restrictions which need to be respected. Technical progress in electronics and sensor technologies as well as a higher demand on the market will help increase the performance of affordable system components, suitable for aerial vehicles that are part of a collective system.

\subsubsection{Localisation and Navigation}

Once the flying platform is stabilised, the next step is to control its displacement. This is often referred to as "navigation" in the robotics literature. The most common approach is to rely on some kind of localisation means in order to follow a predetermined path using a flight controller. In this section, we survey the various approaches that can be used to solve both the problem of localising a flying robot and controlling its path. Advanced techniques of localisation and navigation relying on the presence of cooperating neighbouring agents will be further described in Secs. 17.3 and 17.4.

In outdoor settings, the localisation problem is commonly solved using GPS, which typically provides horizontal precision within a few meters and vertical precision of a few tens of meters. The precision can be improved using enhanced GPS techniques such as Wide-Area Augmentation System (WAAS) or Differential GPS (DGPS), and / or by integrating GPS with an Inertial Measurement Unit (GPS-IMU integration) (Dudek and Jenkin, 2008).

The most common implementations of UAV navigation systems are based on a 


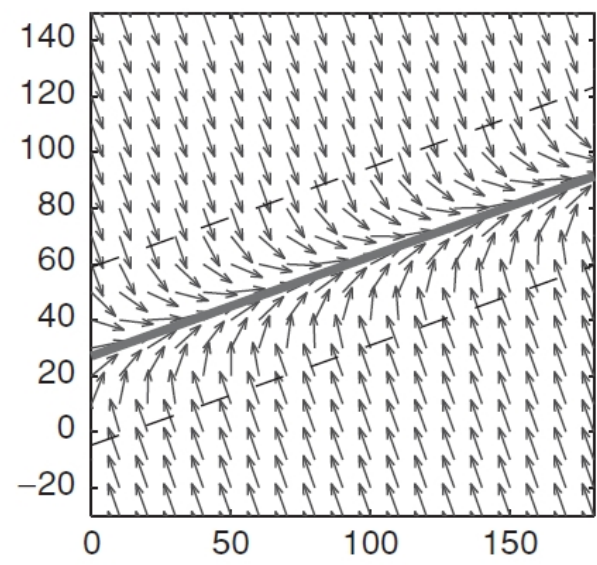

(a)

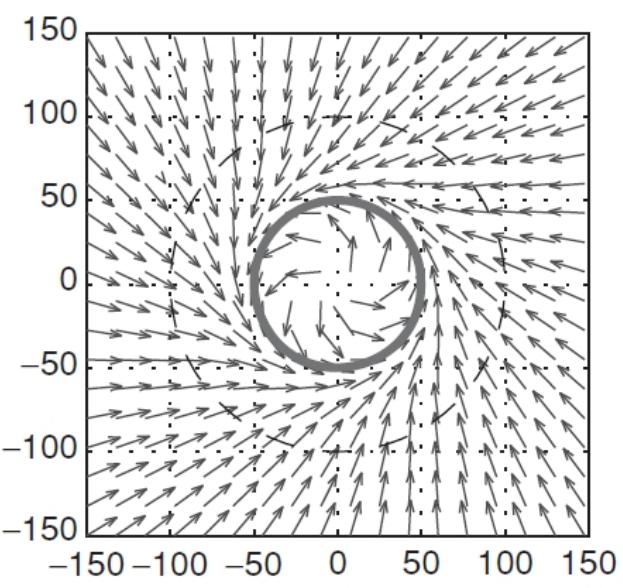

(b)

Figure 17.7. Vector fields producing the desired heading at any position in space to guide a UAV stably onto a desired trajectory element. Shown are examples a) for a straight line and b) for a circular loiter pattern. Combinations of both can be used to form entire flight paths. Adapted from Griffiths et al. (2006).

list of GPS-waypoints which define a flight path. If time constraints are imposed for passing at specific locations, following a flight path becomes a "trajectory tracking" problem (Nelson et al., 2007). In order to assemble a path by its elements between waypoints, Guglieri et al. (2008) suggested an approach based on polynomial splines that respect dynamic constraints of the UAV platform and a continuity condition at each waypoint for smooth transitions. Once the waypoints and path elements are planned, a suitable path tracking algorithm must be found. An example of a stable non-linear tracking controller is presented by Ambrosino et al. (2006). As an elegant way to determine a suitable flight path and a vehicle guidance law at the same time, Lawrence et al. (2008) proposed a vector field technique that is shown to have Lyapunov stability properties. The vector field solution produces simple and stable tracking of trajectories of any shape in 3D, from straight lines to circular loiter patterns. The tracking controller's output is typically the desired heading that steers a UAV asymptotically towards the desired flight path (Fig. 17.7.). The heading error determined with a GPS or compass sensor is then used to command the heading and roll control loops of the autopilot. However, to compensate for the effect of wind, often course (measured again with a GPS) instead of heading is taken into account (Griffiths et al., 2006).

Indoor autonomous operation of aerial robots cannot utilise GPS for positioning or state-estimation due to attenuated signals and multi-path reflections (Siegwart and Nourbakhsh, 2004; Zeimpekis et al., 2003). Absolute positioning indoors has instead been implemented using colour vision cameras (Hoffmann et al., 2008) or with infrared 3-D motion tracking cameras, e.g. the Vicon system ${ }^{1}$, as demonstrated in the works by Ducard and D'Andrea (2009), Oyekan and Hu-

\footnotetext{
${ }^{1}$ www.vicon.com
} 
osheng (2009),Lupashin et al. (2010),How et al. (2008) and Valenti et al. (2007) (see also Fig. 17.5.). Such tracking systems provide high-accuracy $(1-5 \mathrm{~mm})$ measurements of position and attitude at high refresh rates $(200 \mathrm{~Hz})$. This has facilitated the control of small indoor aircraft in highly dynamic manoeuvres such as multi-flip trajectories (Lupashin et al., 2010). However, this technology requires a priori installation of external sensors or beacons, which is infeasible for many applications, e.g in disaster situations.

An alternative approach that has been proposed by Stirling et al. (2010) involves utilising an entirely on-board sensing solution. Robots are equipped with relative-positioning sensors (Roberts et al., 2009), detailed in Sec. 17.3.2. Under collective operation, some robots remain static as beacons, either attached to the ceiling (Roberts et al., 2008) or on the ground. Beacons provide static reference points to flying robots, which can use the relative-positioning information to directly control flight stability and aid navigation. This facilitates truly autonomous operation in unequipped indoor environments, exploiting the power of a collective system to overcome the limited sensing of indoor flying robots.

Another approach to the localisation problem in GPS-denied environments is Simultaneous Localisation and Mapping (SLAM), which is a technique to create a map of an unknown environment while at the same time keeping track of the current robot pose (Bailey and Durrant-Whyte, 2006; Durrant-Whyte and Bailey, 2006). This facilitates navigation (Meyer and Filliat, 2003), stabilisation (Grzonka et al., 2009) and mission-planning for tasks such as search and exploration (Achtelik et al., 2009). SLAM is particularly appealing for indoor aerial robotic operation environments because of the ability to localise the robot without requiring external beacons. Currently, only single robots have been shown to autonomously fly in unknown environments while constructing and maintaining their own map using a SLAM algorithm. For example, Steder et al. (2008) demonstrate real-time monocular and stereo visual SLAM on a variety of small aerial platforms (blimps and co-axial helicopters) for indoor navigation with a 5-8\% error in relative positioning estimates. Alternatively, Grzonka et al. (2009) present a navigation system for a small indoor quadrotor equipped with a laser scanner. Similarly, Achtelik et al. (2009) use a quadrotor with a laser scanner combined with a stereo camera system. The quadrotor can construct a map of the environment, localise itself and search for objects. Presently, these approaches require off-board processing due to the computational complexity of the map construction and maintenance. To mitigate such problems methods for improving the computational efficiency for aerial robots are being developed. For example, Törnqvist et al. (2009) present a SLAM approach based on Rao-Blackwellized Particle Filters and the FastSLAM algorithm (Thrun et al., 2004) for rapid computation. However, so far their work was only evaluated with real-flight data processed off-line. Finally, real-time SLAM has been demonstrated on-board on large outdoor UAV platforms where there is sufficient payload for high-powered computers. For instance, Artieda et al. (2009) demonstrate visual 3-D SLAM on a UPM-Colibri I helicopter with a $12 \mathrm{~kg}$ payload. Similarly, Kim et al. (2007) present results with a fusion of inertial and visual SLAM ran on-board in real-time on a Brumby MKIII UAV with $20 \mathrm{~kg}$ payload. 
In the near future, the collective operation of such strategies will be applied to multiple cooperating flying robots as researchers continuously apply state-of-theart techniques from ground robots to aerial robots and as the on-board sensing and processing capabilities improve and decrease in weight.

\subsubsection{Collision Avoidance}

One of the big issues in aerial systems that is more tricky to solve than in most terrestrial robots is the collision avoidance problem. This is due to the fact that aerial robots evolve in 3D, often have complex dynamical constraints and possess only limited available payload that can be devoted to exteroceptive sensors. Moreover, the sensor coverage often needs to be $3 \mathrm{D}$ as well unlike terrestrial robots where only planar coverage is often sufficient. This section covers possible approaches to static obstacle avoidance. By static obstacles we understand objects such as buildings, trees or the terrain itself. Mid-air collision avoidance between aerial vehicles requires relative positioning techniques or active cooperation, which will be tackled in Secs. 17.3.2 and 17.4, respectively.

Collision avoidance systems require both an obstacle sensing technique and a collision avoidance algorithm, which makes use of the information provided by the sensors to determine a collision-free trajectory. Sensors used for collision avoidance can be classified into passive and active. Passive sensors includes vision systems such as standard cameras, stereo vision or optic flow detectors. Active sensors include triangulation-based range finders (laser or collimated infrared LED), time-of-flight devices such as ultrasonic sensors or radars. In general active sensors are heavier and consume more power because they need to emit signals into the environment. This is often a drawback for small flying platforms that have stringent payload constraints. However, passive sensors are dependent on the energy (e.g. light) available in the environment, which may limit operation in low lighting conditions.

Collision avoidance methods can be roughly classified as reactive or modelbased. Reactive algorithms only take into account the current information provided by the sensors to decide on the action to take in the next time step. Modelbased approaches try to maintain maps of the surrounding environment and rely on path-planning techniques. In general reactive approaches are more adapted to small systems that have limited computational power and tend to have faster dynamics. However, the resulting trajectories cannot be shown to be optimal, which may be problematic in densely cluttered environments.

In order to solve the problem of collision-free navigation in indoor environments, some groups have been looking at active distance sensors. Miniaturisation of ultrasonic, infrared and laser range finders is getting to a point where their mass becomes compatible with indoor flying platforms (Bouabdallah and Siegwart, 2007; Grzonka et al., 2009; Matsue et al., 2005). However, these embedded sensors still lack in accuracy, wide field coverage and refresh rate. More recently though, Achtelik et al. (2009) have been able to fit a planar scanning laser range finder together with a stereo camera system on an indoor helicopter to achieve simultaneous localisation and mapping of the environment, which in turns enable 
collision-free path planning. However, this computationally expensive approach could not be implement on-board the flying robot, but rather on a series of groundbased laptop computers. At the other extreme in terms of computational requirements, we find approaches inspired by flying insects where researchers have been looking at reactive control strategies mainly relying on optic-flow (Zufferey, 2008). These enable impressive miniaturisation of the whole flying robot such that a 10gram platform could be demonstrated to fly completely autonomously in an officesized room (Zufferey et al., 2009). However these approaches using optic flow still have difficulties coping with poorly textured environments.

In outdoor settings, only few people looked at flying in the vicinity of the ground where the robot has to take into account the presence of buildings or uneven terrain. Scherer et al. (2008) demonstrated flight among buildings with a 75-kg unmanned helicopter using a model-based technique together with an active 3D laser range finder, which provided sufficiently accurate data to build and maintain a local 3D map of the surroundings. Another approach using an active radar weighing 300 grams together with a reactive collision avoidance algorithm was implemented on a flying wing by Viquerat et al. (2007), but with only limited results. Griffiths et al. (2007) have been using a combination of a single ray, forward pointing, laser range finder together with a couple of laterally-oriented optic flow detectors (Fig. 17.8.) to demonstrate single building avoidance or biasing of planned trajectory to better follow the centre of a valley. Interestingly, the active laser sensor with its 170 grams was at least one order of magnitude heavier than the passive optic flow detectors. Pushing one step further the optic flow approach, Beyeler et al. (2009) have shown that a simple reactive control strategy together with a set of seven mouse sensors arranged in a conical way around the forward direction could allow a small fixed-wing aircraft (Fig. 17.9.) to stabilise its flight while avoiding collisions with the terrain and trees. One of the limitation of optic flow techniques, though, is that they often assume static obstacles so that the optic flow signals are only generated by the motion of the flying robot and not by that of the surrounding objects.

\subsubsection{Power Management and Energy}

Truly autonomous systems must manage their own energy resources in order to complete missions successfully (Kubo and Melhuish, 2004; O'Hara et al., 2006). However, complete energy autonomy is one of the greatest challenges in mobile robotics, (Mei et al., 2005; Rybski et al., 1998). This is especially true with aerial robots, which typically have a limited flight autonomy of only 10-15 minutes (Roberts et al., 2008; How et al., 2008). Despite the fundamental importance of power management on aerial robots, this area has received relatively little research focus (How et al., 2008). A vast majority of small aerial robots derive power from on-board batteries, therefore energy is finite and unreliable (Wang et al., 2008). Typically, lithium-polymer cells are utilised, which offer the highest commercially available energy-density that facilitates economical recharging. Larger UAVs are often powered by gasoline, which has a much higher energy density providing a longer endurance of up to several hours. However, combustion engines are typi- 


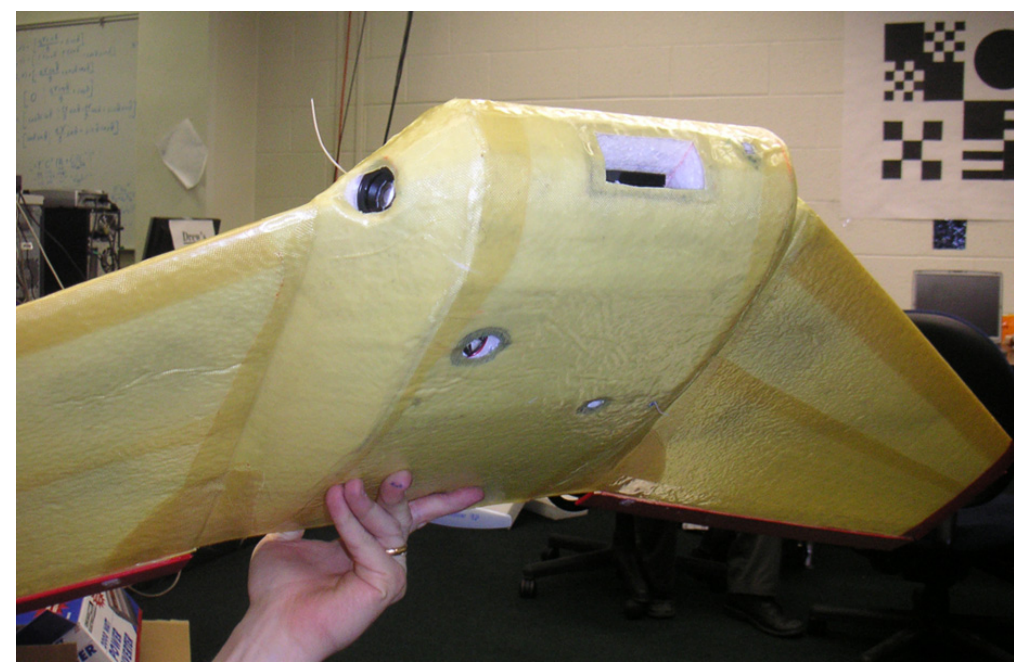

Figure 17.8. The $150 \mathrm{~cm}$ platform used for autonomous flight in canyons and urban environments. The square hole in the centre is for an Opit-Logic RS400 laser range finder (400 m range, $170 \mathrm{~g}$ ), and the circular holes are for the Agilent ADNS2610 optic flow sensors. Image courtesy of Randy Beard, BYU Magicc Lab, Brigham Young University, US.

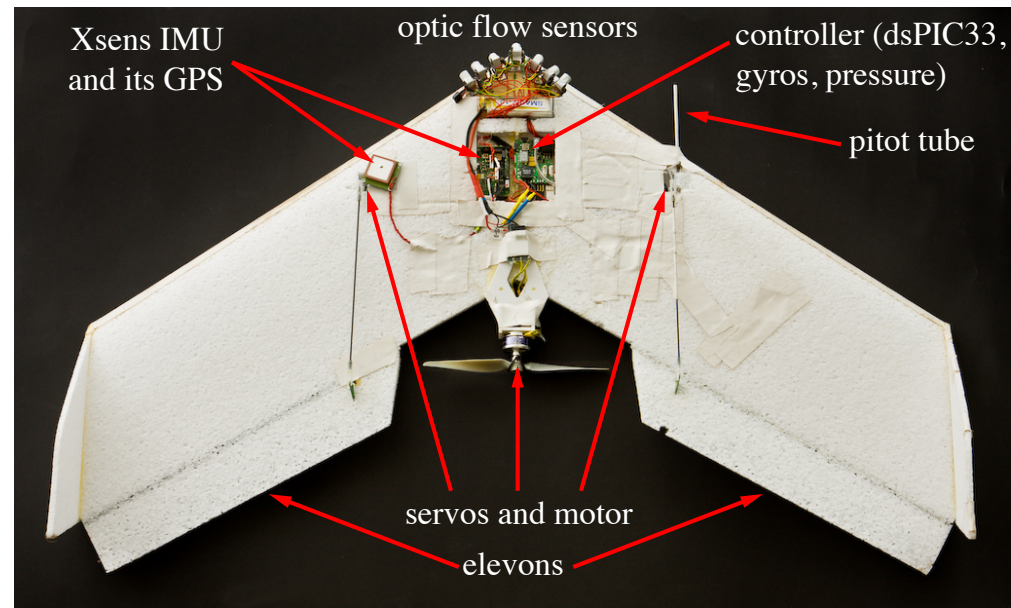

Figure 17.9. The $80 \mathrm{~cm}$ flying wing equipped with a set of seven optic flow sensors (Agilent ADNS5050), which demonstrated robust low-altitude terrain following and collision avoidance using the so-called optiPilot control strategy.

cally heavier and louder than the electric counterparts. The fuel is also an inherent danger rendering them undesirable for indoor operation. In the future fuel-cell technologies (Kundu et al., 2007) or new batteries may provide higher energydensities and increased endurance, however, there is not likely to be any revolutionary changes in the foreseeable future. Therefore, it is important to maximise the efficient usage of the available on-board energy and to examine other means of mitigation.

Common to all aerial robot developments in an attempt to maximise flight en- 
durance is the minimisation of weight by clever mechanical-structural engineering, utilisation of modern materials such as carbon-fibre and composites, reduced electronics weight and the use of MEMs technology, and the optimisation of airfoils, mechanics and rotors. Once the system is optimised alternative methods are sought to prolong endurance. One such approach is to harvest energy from the environment. A promising idea is to use solar cells, as proposed by Noth $e t$ al. $(2006 \mathrm{a}, \mathrm{b})$. In this work, a $2.5 \mathrm{~kg}$ plane with a $3.2 \mathrm{~m}$ wingspan was fitted with 216 GWE-32 silicon cells covering $0.512 \mathrm{~m}^{2}$ which provides $84 \mathrm{~W}$ of power under optimal solar conditions. This was sufficient to fly continuously day and night, using excess solar power in the daytime to charge batteries that power the vehicle through the night. However, the extensive analysis showed that such a system scales poorly to smaller sizes preventing application on lighter aircraft. Also the current system is limited to the times of year, latitudes and weather where it can achieve such continuous autonomy. Moreover, there is little spare payload for taskrelated sensors and processors. Additionally, solar power is of extremely limited use indoors. In general, alternative forms of energy harvesting, even simply locating electric power sockets for instance, is prohibitive since recharging batteries takes several hours, undesirable in most applications such as disaster mitigation.

An alternative idea to harvesting electrical energy is to directly exploit the energy available in the environment. For example, Allen and Lin (Allen and Lin, 2007) propose to exploit thermals, similar to many large birds and glider pilots, to gain lift and thus altitude. This increases range of operation and the gained altitude also acts as stored kinetic energy. In this work the autonomous glider gained on average $172 \mathrm{~m}$ of altitude over 23 trials. However, this approach is strongly dependent on environmental conditions and is limited to outdoor operation.

For situations where it not possible to harvest energy, one method to prolong mission endurance for many tasks such as surveillance and monitoring is to employ perching techniques so that the aircraft can remain at rest. This is commonly demonstrated in nature where birds perch on branches conserving energy while maintaining the elevated viewing position and safety by being off the ground. Researchers are now applying such techniques to aerial robots (Desbiens et al., 2009; Roberts et al., 2008; Kovac et al., 2010). Perching can be especially advantageous in indoor environments where there are many naturally occurring perching features. However, implementation difficulties include the mass of the attachment and detachment mechanism, mechanical design complexity, and perception of safe and amenable perching locations. In (Roberts et al., 2008), the attachment mechanism was simplified by using magnets to attach to ferromagnetic ceilings. This work quantitatively showed the gain in mission endurance possible with such perching techniques. Perching also offers the benefits of stable long term sensing which can be utilised to provide cleaner sensory information. For example, while being statically attached to a ceiling a robot can capture images without vibrations using long exposure times to retrieve low-noise images without motion blur. Lastly, when developing collective aerial systems with perching abilities it is important to be able to properly exploit this ability to gain energy efficiency in cooperative tasks. For example, (Stirling et al., 2010) showed how perching can be exploited 
within a swarm of aerial robots within a search task. Results indicate that 3-4 $\times$ the energy was saved using the perching capabilities rather than maintaining a static hover.

Finally, an important method of increasing energy autonomy is from efficient behavioural operation, both individually but especially in collective systems. For example, motion paths can be optimised to reduce energy costs (Mei et al., 2004; Wang et al., 2008), and formation flight may reduce power consumption by decreasing induced drag (Chichka and Speyer, 1998). Furthermore, avoiding duplication and interference in collective systems is vital for efficient operation (Rosenfeld et al., 2006; Rybski et al., 1998) and has only just started to be researched in aerial systems (Stirling and Floreano, 2010b,a).

\subsection{Enabling Coordination}

In this section we discuss the necessary technologies to allow collective operation with aerial robots. Multirobot applications usually entail cooperative operation by coordinating individual actions harmoniously. Coordination in collective systems is paramount to the efficient exploitation of available robot resources. In order to achieve coordination two key technologies are usually required: communication and relative positioning. Communication allows the coordination of behaviours and the efficient transfer of information. Relative positioning allows the spatial coordination of proximal robots to achieve behaviours such as collision avoidance, formation flight, or optimal dispersion for sensor coverage. Although these technologies are often distinguished by hardware, software and functionality, hence the separation here, one should note that for many operations the two are inseparable. Relative positioning systems often provide, or even require, communication capabilities, while communication hardware can be exploited to provide relative positioning information.

\subsubsection{Communication}

Wireless communication is a requirement in most systems involving flying robots. It creates a vital link between the human operator and the flying robot, it also allows for inter-robot communication which is important for collective systems. In real-world applications, robots need to be able to explore large warehouses or cover outdoor areas of several square kilometres. Therefore, they will need to have efficient long-range communication so that the robots can spread-out sufficiently in their environment to be useful.

There are currently many wireless devices available, each is suitable for a variety of different applications. Some are designed to transmit at high data-rates, like Wireless USB and WIFI, others are designed to transmit over long distances, like GSM and radio modems, and some can do both, like WiMax (Table 17.1). However, to select a suitable wireless device for a particular application there are some important constraints and considerations.

By using the free space path loss model (Seybold (2005)) it is possible to esti- 
mate the power loss over a distance, and therefore the minimum required transmitter power and antenna combination to create a line-of-sight wireless link:

$$
L_{F S}(d B)=20 . \log _{10}(D)+20 . \log _{10}(F)-27.55
$$

From this equation we can see that the free space power loss, $L_{F S}$ in $d B$, depends on the operational link distance, $\mathrm{D}$ in meters, and the frequency, $\mathrm{F}$ in $\mathrm{MHz}$. We can then apply this to calculate the link budget with different types of transmitter, receiver and antenna combinations.

$$
L_{F S}=P_{T X}+G_{T X}-L_{T X}+G_{R X}-L_{R X}+P_{R X}-L_{M}
$$

The link budget is simply a sum of all the components of the RF power system, with positive and negative contributions. Positive contributions include the sensitivity of the receiver, $P_{R X}$ in $|d B m|$, the transmitter output power, $P_{T X}$ in $d B m$, the transmitter and receiver antenna gains, $G_{T X}$ and $G_{R X}$ respectively in $d B i$. Negative contributions include the free space power loss, $L_{F S}$ in $d B$, link margin, $L_{M}$ in $d B$, the cable/ connector loss, $L_{T X}$ and $L_{R X}$ in $d B$.

Lets take a typical WIFI link as an example. We can re-arrange Eq. 17.2 so that the link distance, D, can be determined from the specific hardware parameters.

$$
D(m)=10^{\frac{\left(L_{F S}-20 . \log _{10}(F)+27.55\right)}{20}}
$$

Lets set-up a system that has two off-the-shelf $50 \mathrm{~mW}$ WIFI devices. Both devices have a transmitter power of $17 \mathrm{dBm}(802.11 \mathrm{~g})$ and a receiver sensitivity of $-70 \mathrm{dBm}$ (@54 Mbps). The device on the flying robot has an omni-directional antenna which is connected directly to the device, and the ground station has an omni-directional antenna connected using a cable with a loss of $2 d B$, both antennas having a nominal gain of $2.2 \mathrm{dBi}$. The link margin should be high enough to make sure the link is robust to small deviations from the estimation, so lets set this to a nominal value of $10 \mathrm{~dB}$. If we apply these parameters to Eq. 17.4, we get a link distance of $\approx 93 \mathrm{~m}$, which matches what we see in Table 17.1. Interestingly, if we substitute the antenna on the ground station to a Yagi directional antenna with a gain of $13.5 \mathrm{dBi}$, then we can see that the link distance increases to $\approx 340 \mathrm{~m}$, which is an increase of $247 m$ simply by changing the antenna.

Therefore, in order to increase the operational link distance, the transmitter power must increase and the use of higher gain antennas is required to improve signal strength. However, increasing the transmitter power means that the amplifier needs to be larger, to dissipate more heat, meaning an increase in power consumption, size, weight and cost, also there are regulations that limit the legal maximum power. We can also see that if the frequency is reduced then the path loss also reduces. However, this would also reduce the available bandwidth.

The size of the antenna generally relates to the amount of energy it can collect/emit and can be expressed as a function of antenna aperture area (A), wavelength $(\lambda)$ and efficiency $(\eta)$ (Lo and Lee, 1993).

$$
\operatorname{Gain}(d B i)=10 \cdot \log _{10}\left(\frac{4 \cdot \pi \cdot \eta \cdot A}{\lambda^{2}}\right) .
$$

Therefore, a larger antenna means better performance. Note that if the wavelength is reduced (i.e frequency increased) then the gain of the antenna increases. This means that as the frequency increases the size of the antenna decreases. 
The radiation pattern of an antenna is important to determine the best antenna for the application. The shape of the radiation pattern of an antenna determines how the transmitter power is radiated with respect to the centre of the antenna. An antennas radiation pattern can be plotted as a graphical representation of the intensity of the radiation $v s$. the angle from the perpendicular of the antenna (Balanis, 1997). The directivity, or beam-width, of an antenna is determined by measuring at which angle the power level falls to half power i.e. $3 \mathrm{~dB}$ below the maximum point. This means that a highly directive antenna, with a narrow beam-width, will condense the transmitter power in a smaller angle and increase the gain of the antenna in the corresponding direction.

Generally, due to the high mobility of flying robots, a vertically aligned omnidirectional antenna is required. Omni-directional antennas, like a $1 / 2$ wavelength dipole (Lo and Lee, 1993), usually have a radiation pattern like a doughnut (Fig. 17.10.) where the directivity of the maximum gain covers a full 360 degrees. This means that the flying robot can change its direction without affecting the wireless link.
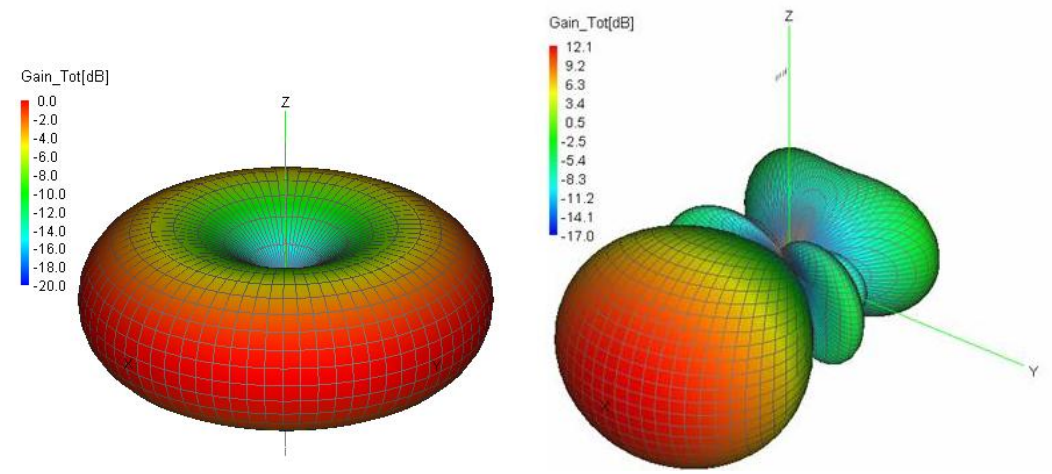

Figure 17.10. Left: 3-D radiation pattern of a dipole omni-directional antenna, right: 3-D radiation pattern of a yagi directional antenna (www.antenna-theory.com, accessed 14/04/2010).

One consideration here is that the doughnut shape has two null zones, on the top and bottom. Therefore, if the robot is directly above another robot or a ground station, or the antenna is tilted, the wireless link may be lost. Alternatively, a high gain directional antenna, like a yagi (Fig. 17.10.), on the ground station, which is directed towards the omni-directional antenna on the flying robot, can be used to achieve a longer link distance. However, due to the higher directionality, the antenna may need to be dynamically aligned as the flying robot's path changes.

When considering wireless communication hardware there is a compromise between operational distance, power consumption, data-rate, size and weight of the communications device and its antenna. These are characteristics that need to be optimised for a given application, which is not a trivial task and often leads to acquiring a solution to suit a very specific problem or application.

As well as hardware constraints, with flying robots special considerations must be employed to create robust network topologies, protocols and packet routing 
systems. In particular, algorithms to route packets through a network of flying robots must be highly reactive to breaks in the network by rapidly recalculating new routes (Timcenko et al., 2009). Alternatively, the network can be tolerant to breaks by having robots store messages which cannot be transmitted until a route has been established (Shen et al., 2008). Interestingly, the high speed at which flying robots can change position also allows them to rapidly reposition themselves so as to optimise communication or repair breaches in the network (Basu et al., 2004; Dixon and Frew, 2009; Hauert et al., 2010b).

With all this in mind, due to the limited payload available on a flying robot, it is hard to find wireless hardware that meets the strong weight, size and energy constrains. Most researchers working with flying robots will opt for radio modems as they are light weight, medium power devices (less than $1 \mathrm{~W}$ ) that have a good range capability (up to $100 \mathrm{~km}$ ). This longer range is traded for a lower bandwidth, typically up to $250 \mathrm{Kbps}$. This lower bandwidth introduces a problem for collective robotics as it must be shared with the other nearby robots. To solve this problem many researchers working on collective robotics are compromising the longer range of radio modems for higher bandwidth devices (Hauert et al., 2010b; Hoffmann et al., 2004; De Nardi et al., 2006). It has become the new trend to use WIFI devices as they are small, cheap and have become easily available (Ahrens et al., 2009; Cheng et al., 2006; Kendoul et al., 2009; Lizarraga et al., 2008). However, due to the increase in portable wireless consumer products, we are already faced with the problem of frequency saturation of the allocated spectrum (Berne and Pogorel, 2003) and this is only going to get worse.

With the boost in the mobile phone industry in the last decade, GSM and 3G mobile data services have become an alternative to using license-free amateur frequency bands (Brisset and Hattenberger, 2008; Tozer et al., 2000; Wzorek et al., 2006). Advantages include global coverage, reasonable bandwidth (up to $14 \mathrm{Mpbs}$ ) and small antenna requirement. With special connection rates and capped plans it can even be reasonably inexpensive. However, the power requirement is relatively high (less than $2 \mathrm{~W}$ ), there are dead spots in remote locations and the hardware is usually larger than other wireless devices.

In summary, enabling wireless communication for aerial collective robotics inparticular, requires a trade-off between operational distance, power consumption, bandwidth, size and weight of a device. Careful choices must be made to select the best network topology, protocol and packet routing for real-world applications.

\subsubsection{Relative Positioning}

For many applications in aerial collective robotics, individual robots are required to have knowledge about the relative distance and bearing to neighbouring robots. Relative positioning is crucial for individual decision making, formation control and coordination, e.g. (McLurkin and Smith, 2007; Payton et al., 2004). For instance, knowledge about the relative distance to other robots can provide a cue about the risk of breaking a communication link. Knowledge of the bearing can provide a cue about the risk of collisions when pursuing a given trajectory (Carnie 
Table 17.1. Comparison of wireless communication devices

\begin{tabular}{|c|c|c|c|c|c|}
\hline Common Name & $\begin{array}{l}\text { Range }(\mathrm{m}) \\
(† \text { omni-directional }) \\
(\star \text { directional })\end{array}$ & $\begin{array}{l}\text { Bandwidth } \\
\text { (Mbps) }\end{array}$ & $\begin{array}{l}\text { Tx Power } \\
\text { (W) }\end{array}$ & Limitations & $\begin{array}{l}\text { Frequency } \\
\text { (MHz) }\end{array}$ \\
\hline $\begin{array}{l}\text { Wireless } \\
\text { USB }\end{array}$ & $<10+$ & $<480$ & $<0.3$ & $\begin{array}{l}\text { Short range, } \\
127 \text { devices }\end{array}$ & 3,100 to 10,600 \\
\hline $\begin{array}{l}\text { Bluetooth } \\
\text { IEEE802.15.1 } \\
\text { IEEE802.11 }\end{array}$ & $<100+$ & $<3$ & $<0.1$ & 7 devices & 2,400 \\
\hline $\begin{array}{l}\text { WIFI } \\
\text { IEEE802.11a } \\
\text { IEEE802.11b } \\
\text { IEEE802.11g } \\
\text { IEEE802.11n }\end{array}$ & $\begin{array}{l}<30+ \\
<90+ \\
<90 \dagger \\
<250+\end{array}$ & $\begin{array}{l}54 \\
11 \\
54 \\
600\end{array}$ & $\begin{array}{l}<0.1 \\
<0.1 \\
<0.1 \\
<1\end{array}$ & $\begin{array}{l}\text { Spectrum } \\
\text { saturation } \\
(2,400 \mathrm{MHz})\end{array}$ & $\begin{array}{l}5,000 \\
2,400 \\
2,400 \\
2,4005,000\end{array}$ \\
\hline $\begin{array}{l}\text { Zigbee } \\
\text { IEEE802.15 }\end{array}$ & $<1,600 \star$ & $<0.25$ & $<0.1$ & $\begin{array}{l}\text { Low } \\
\text { bandwidth }\end{array}$ & $\begin{array}{l}868,915, \\
2400\end{array}$ \\
\hline $\begin{array}{l}\text { GSM 07.05/.07 } \\
\text { EDGE, HSPA+ }\end{array}$ & $\begin{array}{l}<35,000 \dagger \\
\text { (network) }\end{array}$ & $\begin{array}{l}<0.27 \\
<14\end{array}$ & $\begin{array}{l}<2 \\
<2\end{array}$ & $\begin{array}{l}\text { Service Charge, } \\
\text { dead zones }\end{array}$ & $\begin{array}{l}850,900,1,800, \\
1900,2,400\end{array}$ \\
\hline $\begin{array}{l}\text { WiMax } \\
\text { IEEE802.16d/e }\end{array}$ & $<50,000 \star$ & $<70$ & $\begin{array}{l}<20 \text { (base) } \\
<0.3 \text { (node) }\end{array}$ & $\begin{array}{l}\text { High power } \\
\text { consumption }\end{array}$ & $\begin{array}{l}2,300,2,500 \\
3,500\end{array}$ \\
\hline $\begin{array}{l}\text { Radio Modem } \\
\text { IEEE802.15 }\end{array}$ & $<64,000 \star$ & $<0.25$ & $<1$ & $\begin{array}{l}\text { Low } \\
\text { bandwidth }\end{array}$ & $\begin{array}{l}868,915 \\
2,400\end{array}$ \\
\hline
\end{tabular}

et al., 2006). Knowledge of relative positioning can also be used to find an optimal distribution of robots over a given area to maximise the coverage area of sensors, e.g. (Howard et al., 2002). Until recently, most relative positioning systems have been developed for operation only on ground robots (Pugh et al., 2009; McLurkin and Smith, 2007; Payton et al., 2004) that afford simplifying constraints such as operation exclusively on horizontal ground planes. More recently, relative positioning systems for underwater robot swarms that operate in 3-D space have been developed (see Chapter 16).

Considering aerial collective systems, solutions for relative positioning must typically comply with severe constraints in terms of size, power consumption, weight and processing requirements. Specific requirements for aerial systems include 3D operation rather than a 2D-planar system as is typically used for ground robots (Pugh et al., 2009; McLurkin and Smith, 2007; Payton et al., 2004). Importantly, application-dependent issues include increased range, resolution, update rates, scalability, and robustness to environmental interference.

One possibility to obtain relative positioning information is to spread global positioning information (see Sec. 17.2.3) over a communication network (see Sec. 17.3.1), to which all robots are connected, e.g. (York and Pack, 2008; DeLima et al., 2006). This can happen either with a global broadcast from a central entity with global information, or in a distributed manner over local communication links. The GPS-based FLARM ${ }^{2}$ system, used to calculate the relative position between small manned aircraft for collision avoidance purposes, is an example of local broadcasts of global positioning information (over ranges of about $4-8 \mathrm{~km}$ ). However, robots may be denied access to global position information,

${ }^{2}$ http:/ / www.flarm.de/ 
especially in indoor environments and occluded outdoor areas. Furthermore, even if this information is available, its transmission requires functional communication links and active cooperation. Moreover, global positioning systems usually rely on expensive infrastructures, are vulnerable to interference and jamming, and may have unsatisfactory resolution and update rates. In the case of a centralised broadcast of global positioning information, there is the additional disadvantage of non-scalability due to communication bandwidth limits. Therefore, relative positioning without sharing global positioning information is an important research aspect in collective robotics. Here we summarise current sensor technologies to perform on-board relative positioning, with a distinction between indoor and outdoor applications.

Outdoor Relative Positioning Research in outdoor relative positioning has mainly looked at passive electro-optical sensors (cameras, infrared detectors), passive acoustic sensors and active microwave sensors (RADAR). Also, several researchers have investigated the use of on-board communication hardware as positioning devices.

Utt et al. (2005) developed a monocular vision system based on CCD cameras to detect the motion and track the position of another aircraft relative to a background scene (Fig. 17.11. a)). The real-time passive system provides high radial resolution (0.5 milli-rad) and a horizontal field-of-view of $90^{\circ}$ in front of the vehicle, but vertical field-of-view and ranging capabilities are not specified. Also, sensitivity to vibrations and weather conditions lead to false positives, which can be ruled out in software only at the cost of higher system latency. Shakernia et al. (2005) specifically investigate the feasibility of better range estimates with optical methods by small self-manoeuvres. However, improvements were found to be restricted to impractical, particular manoeuvres, especially perpendicular to line-of-sight.

Binocular (stereo) vision systems are also documented in literature, mostly based on commercial sensor suites available today. A system that computes the distance from a rotorcraft to a fixed obstacle is analysed in (Byrne et al., 2006). Relative localisation in $1 \mathrm{D}$ (range) is shown to be accurate to about $2 \mathrm{~m}$ at $15 \mathrm{~m}$ distance. However, the field-of-view is restricted by optical constraints of stereo vision systems, which typically do not use wide-angle optics like fish-eye lenses.

Active optical sensors like laser range finders, which rely on the principle of time-of-flight measurements of narrow-beam laser pulses, are the preferred choice in ground-based robots due to their range, accuracy and scanning speed while providing a large field of vision (Thrun et al., 2006). Unfortunately, the weight of these sensors restricts their field-of-use to UAVs with sufficient payload capabilities, like large helicopters, as demonstrated by Scherer et al. (2008).

The use of acoustic sensors has been studied by SARA Inc. ${ }^{1}$ for the "Passive Acoustic Non-cooperative Collision-Alert System" (PANCAS), shown in Fig. 17.12.. Although not very precise for $3 \mathrm{D}$ localisation, the microphone array provides a directional cue for noise being emanated by other aircraft or sources on the ground. Light-weight and computationally inexpensive, the developed system has the advantage of being independent of lighting conditions, but exact performance

\footnotetext{
${ }^{1}$ www.sara.com
} 


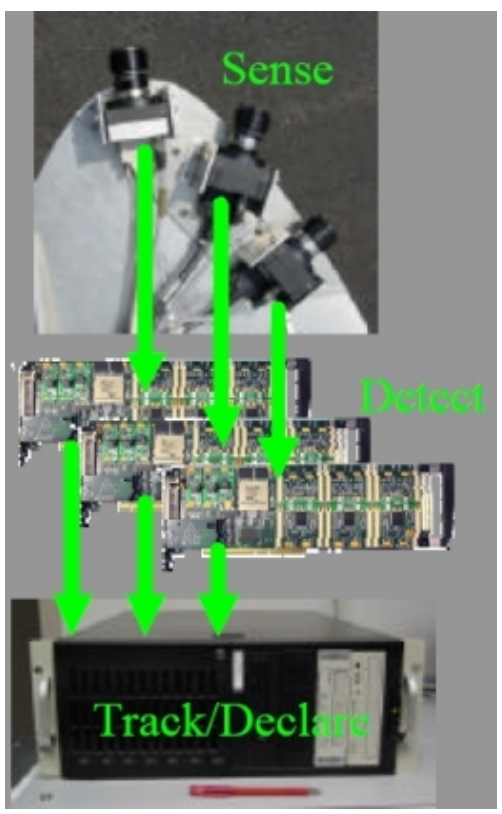

a)

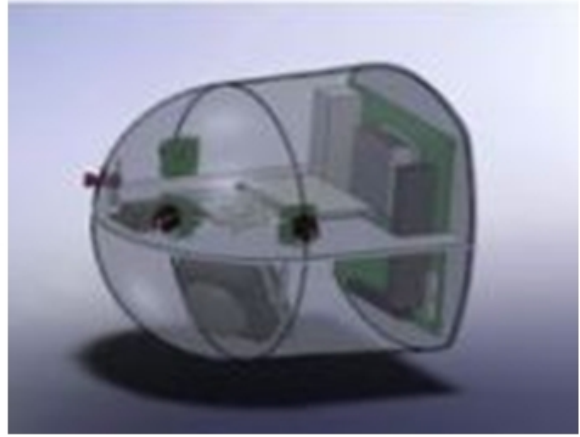

b)

Figure 17.11. Approaches to relative positioning with optical sensors in the context of mid-air collision avoidance: a) Several cameras, integrated in the nose-cone of a small general aviation aircraft, are used in parallel to increase the field-of-view and constitute a high-resolution image for the tracking of another aircraft. Reprinted with permission of the American Institute of Aeronautics and Astronautics. b) An obstacle detection and tracking set-up consisting of 4 cameras, a gimbal-mounted radar and a processing unit is under development by AeroSpy Sense \& Avoid Technology (www.aerospy.at, accessed 01/11/2010).

characteristics are undisclosed.

Relative positioning in commercial aircraft is radar-based. The US Federal Aviation Administration (FAA) issues the specifications of the Airborne Collision Avoidance System (ACAS), implemented generally as Traffic Alert and Collision Avoidance System (TCAS) and using a variant of an active radar, the so-called secondary surveillance radar (SSR). It determines not only range and bearing to nearby aircraft, but interrogates all (radar-)transponders in range, which in turn reply with encoded complementary information like the aircraft's identity or its flight altitude. Being bulky $(5-10 \mathrm{~kg})$ and costly, embedding a full TCAS system on-board smaller aircraft or UAVs is currently not feasible.

Radar as a sensor is also proposed by Viquerat et al. (2007) in the form of a Doppler radar to detect the presence of, and the angular direction towards, an obstacle or aircraft. The developed hardware weighs only some $100 \mathrm{~g}$ with a power consumption of $\approx 5 \mathrm{~W}$, but range $(\approx 10 \mathrm{~m})$ and resolution $\left(\approx 15^{\circ}\right)$ are low and the field-of-view of $\approx 30^{\circ}$ (horizontal and vertical) is small. Unlike monocular electrooptical systems, relative motion with respect to another robot or object is not required for detection. However, the achieved low detection range restricts the area 


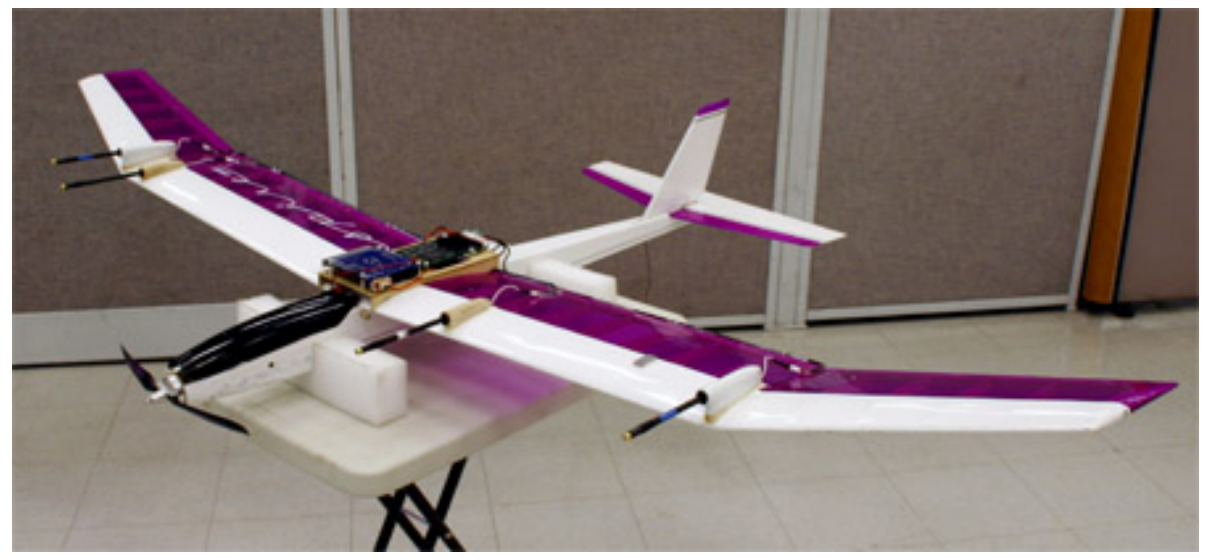

Figure 17.12. The "Passive Acoustic Non-cooperative Collision-Alert System" (PANCAS). A microphone array provides a directional cue to nearby UAVs. The passive acoustic sensors can be seen mounted on the glider's wings. They are smaller, lighter and more energy efficient than optical and radar systems, provide a spherical instantaneous coverage area and work day or night independent of the weather (www.sara.com, accessed 01/11/2010).

of use to collision avoidance with slow moving vehicles.

To overcome the significant disadvantages of particular single exteroceptive sensors, combinations of different sensor technologies were proposed, like adding a radar sensor to a suite of 4 cameras (AeroSpy Sense \& Avoid Technology ${ }^{3}$ ), shown in Fig. 17.11. b). However, data fusion from multiple sensor sources is still work in progress and a functional system could not yet be demonstrated.

An alternative method suggests to extract information about the local radio frequency (RF) environment by measuring the signal-to-noise ratio (SNR) (Dixon et al., 2005). Combined with knowledge of its own motion, a receiving flying robot detects the SNR gradient, which makes it possible to obtain a directional estimate of a transmitting robot's location. The researchers assume omni-directional antennas, radio reception that is independent of robot attitude and orientation, and a smooth SNR environment. However, the analysis was performed in simulation and an evaluation in a real-world experiment has not been reported yet. The mapping of the RF environment experienced by a real flying robot is shown in (Frew et al., 2007).

Some researchers have extensively studied time-of-flight measurements with different commercially available radio communication devices (Lanzisera et al., 2006; Wibowo et al., 2009). Today, dedicated hardware with built-in ToF ranging capabilities exist. Nanotron Technologies $\mathrm{GmbH}^{2}$ proposes "nanoLOC" communication modules with ranging capability. A particular method called "symmetric double sided two-way ranging" permits accurate synchronisation of the internal clocks of linked communication modules, necessary to achieve range resolutions at the meter level. However, the use of these modules has not yet been documented in relation to flying systems.

\footnotetext{
${ }^{3}$ www.aerospy.at

${ }^{2}$ www.nanotron.com
} 
Indoor Relative Positioning In contrast to outdoor aerial robot relative positioning, systems for indoor operation must be more robust to environmental reflections and interference and have stronger size-weight constraints suitable for the lower payloads of smaller indoor platforms. However, often a shorter range is required which facilitates active signal emission as a basis for relative positioning measurements. Many systems exist for ground robots that depend on complex processing with cameras (Nakamura et al., 2003; Spletzer et al., 2001), or other heavy hardware (Montesano et al., 2004). However, these are not suitable for small indoor aerial platforms due to size and weight restrictions and they also often assume a planar distribution of robots.

Ultrasound can be used to achieve high accuracy relative positioning with a light-weight time-of-flight sensor (Rivard et al., 2008; Shoval and Borenstein, 2001), see Fig. 17.13.. However, such active-acoustic sensors have a relatively slow re fresh rate (e.g. $\frac{1}{0.075 \mathrm{~N}} \mathrm{~Hz}$ for $\mathrm{N}$ robots according to Rivard et al. (2008)) due to slow sound dissipation. Therefore, ultrasound is not suitable when large numbers of robots need to be synchronised to avoid interference and sensor crosstalk.

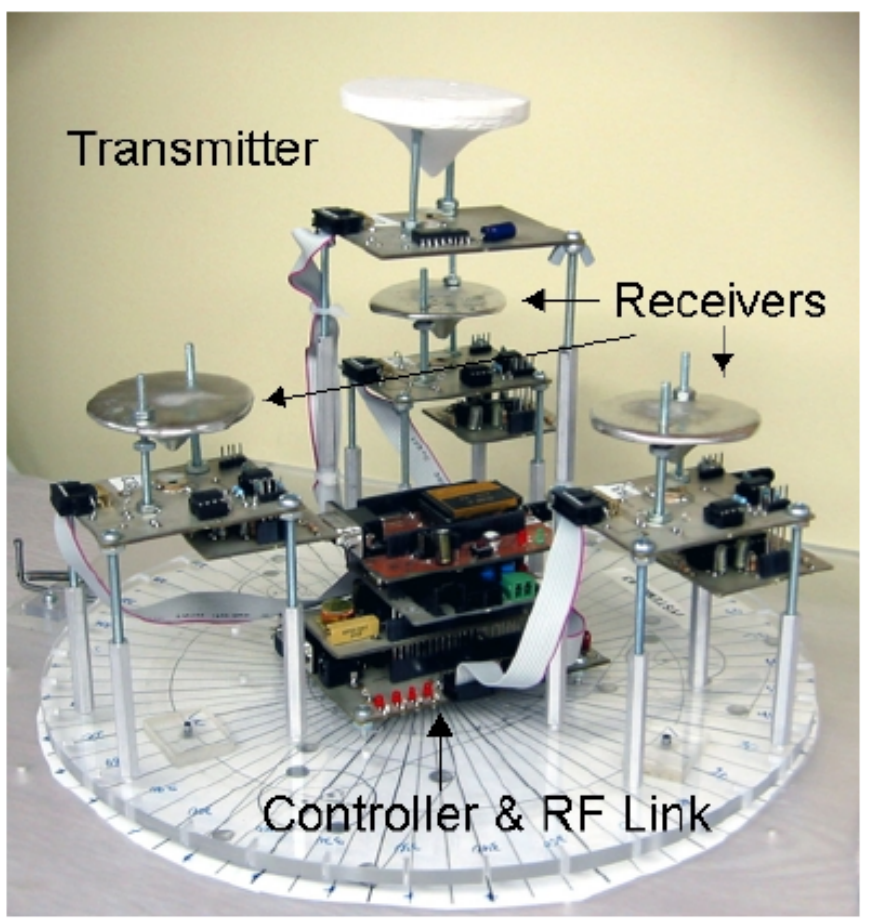

Figure 17.13. A relative positioning system using ultrasound transmitters and receivers based on time-of-flight measurements. This sensor achieves an accuracy of $8 \mathrm{~mm}$ and $3^{\circ}$ with a range of nearly $3 \mathrm{~m}$. Image courtesy of Francois Michaud, Research Laboratory on Mobile Robotics and Intelligent Systems, Univerité de Sherbrooke, Québec.

In contrast, using infrared signals as a medium for relative positioning has the 
benefits of high update rates and small low-cost hardware. The performance of such sensors have been validated in several ground-based multi-robotic systems (McLurkin and Smith, 2007; Payton et al., 2004). The maximum range of these sensors is usually no further than $2.5 \mathrm{~m}$ (McLurkin and Smith, 2007) to $3.0 \mathrm{~m}$ (Pugh et al., 2009), with refresh rates up to $25 \mathrm{~Hz}$ for 10 robots, where the refresh rate is inversely proportional to the number of robots to allow signal dissipation.

Considering the benefits of high update rates and small light hardware, infrared based relative positioning systems are promising for indoor operation. To date there is little research concerning aerial robots. However, Melhuish and Welsby (2002) used an infrared system with a $5 \mathrm{~m}$ range on a swarm of lighter than air vehicles and achieved a simple gradient ascent behaviour. More recently, Roberts et al. (2009) presented a novel infrared relative positioning system suitable for small indoor flying robots. Their system is significantly smaller and lighter, has longer range, higher communication bandwidth, greater immunity to environmental luminosity changes and higher precision than comparable systems developed for ground robots. Importantly for aerial robots, the system is also immune to small differences in heights between robots and attitudinal tilting of the sensor up to 20 degrees, which are important features for aerial systems which do not operate on a level ground-plane. However, so far the system has not yet been demonstrated on flying robots. The novel sensor is shown in Fig. 17.14.

To summarise, currently there has been only limited developments in relative positioning systems for aerial robots and so far there is no universal solution due to the numerous challenges involved. The different constraints between indoor and outdoor operation have so far led to different sensing solutions, even if some technologies are similar. The extended range required for aerial operation, especially outdoors, and the associated increase in power that is required for active sensing approaches exasperates the challenge. Passive sensing, e.g. with visual systems, may mitigate power issues but instead must provide 3D sensing through heavy optical or active mechanical (e.g., pan-tilt) solutions combined with powerful processing. Vision based systems are also affected by illumination issues and may have reduced range. Finally, collective operation spans a broad range of systems from a pair of robots, to large-scale multirobot systems that maintain a centralised or fully synchronised and globally coordinated operation, to entirely distributed swarm systems using only local coordination and pertaining to maximum scalability. These different degrees of cardinality and scalability require different functionality of the relative positioning system such as synchronisation between sensors. Many active sensing approaches, e.g., through ultrasound or infrared signal emission, require turn-taking approaches to differentiate signals, which limits scalability. Furthermore, update-rates are adversely affected by slow dissipation of the source signal. However, if strong coordination is available between robots then they can cooperate in improving the relative positioning information by fusing shared data between robots.

The lack of simple viable relative positioning solution has prohibited its widespread use in research using hardware, but much simulation based research heavily relies upon appropriate relative positioning technology. This dissidence 


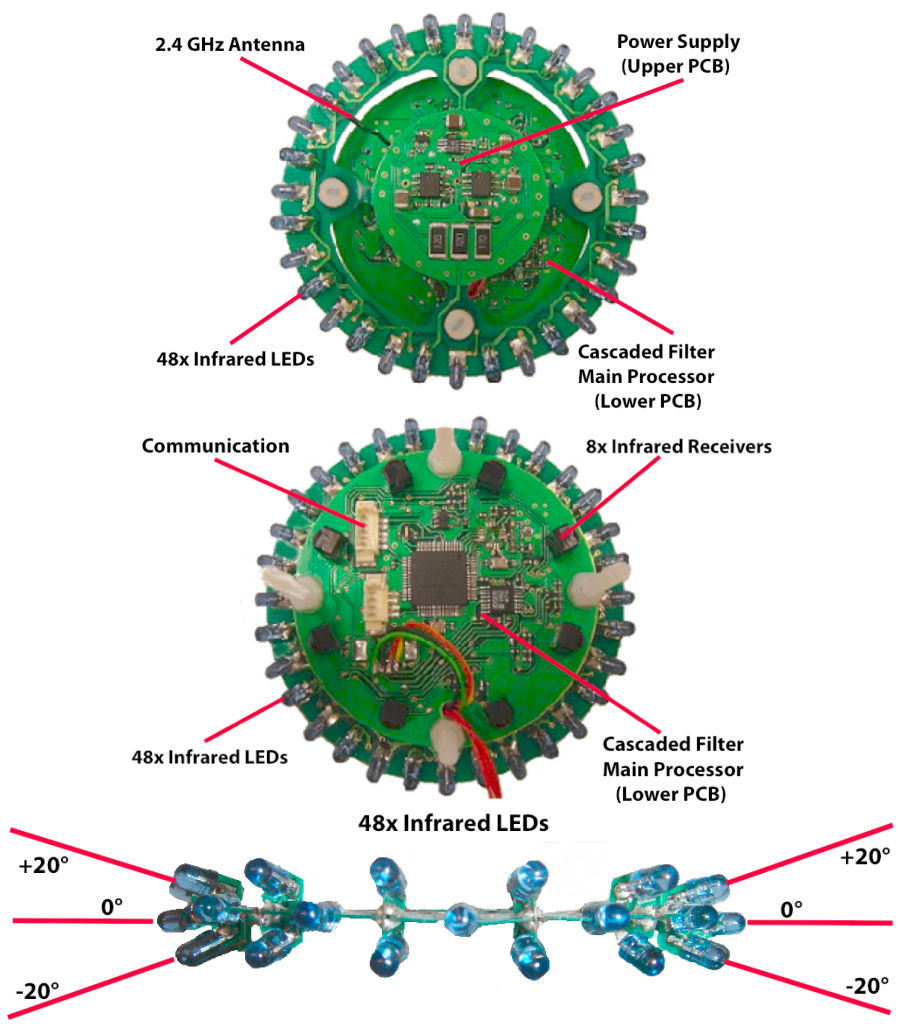

Figure 17.14. The infrared relative positioning system designed for indoor flying robots. The sensor is significantly smaller and lighter than previous infrared relative positioning systems with a diameter of $70 \mathrm{~mm}$ and a weight of only $20 \mathrm{~g}$.

must be acknowledged by researchers working within simulation while we await more advanced hardware solutions.

\subsection{Collective Behaviours}

Groups of flying robots have been envisioned for a wide range of applications from environmental monitoring, surveillance and communication relay to art. Here we propose a classification of the underlining behaviours leading to collective aerial systems into two broad families, namely exploration and flocking. Exploration allows robots to search, monitor, survey and sense their environment by allocating robots to different areas of interest. Flocking on the other hand allows robots to move coherently in groups, with the movement of each agent directly affecting the movement of neighbouring robots. In such systems, robots complete each other, thereby allowing for the distribution of communication, sensing and computational resources across different robots. Throughout this section we will cover these enabling behaviours currently implemented in collective aerial systems and describe the approaches used to design them. Unless stated otherwise, research involving flying robots has been conducted in simulation. 


\subsubsection{Exploration}

Several strategies have been devised to search for areas of interest in an environment with applications in the detection of chemical plumes (Kovacina et al., 2002; Lawrence et al., 2004; Oyekan and Huosheng, 2009; Zarzhitsky and Spears, 2005), wild fires (Gancet et al., 2005; Merino et al., 2006), victims (Kuiper and NadjmTehrani, 2006; Murphy et al., 2008), objects (Stirling et al., 2010) and other targets of interest (Altshuler et al., 2008; Cole et al., 2006; Ruini and Cangelosi, 2009; Sauter et al., 2005; Yang et al., 2005).

The straight forward way to coordinate an exploration task using multiple flying robots is to divide the environment into regions that will each be assigned to a separate robot as proposed by Kovacina et al. (2002). Each robot can be assigned to one area or a set of waypoints by the operator. An example shown in Fig. 17.15. involving five fixed-wing robots was demonstrated outdoors in reality (Allred et al., 2007). Centralised controllers have been envisaged to plan the trajectories of the robots so that the correct robot visits the correct search areas at the correct time while avoiding collisions. The problem is described as a mixedinteger linear program (MILP) that can be solved optimally (Bertuccelli et al., 2004; Richards et al., 2002). Since MILPs and other global optimisation techniques are intractable for large numbers of robots, heuristics and approximations have been developed for similar problems (Alidaee et al., 2009). One noticeable example of optimal path planning with real robots was conducted outdoors with three fixed wing platforms by Beard et al. (2006).

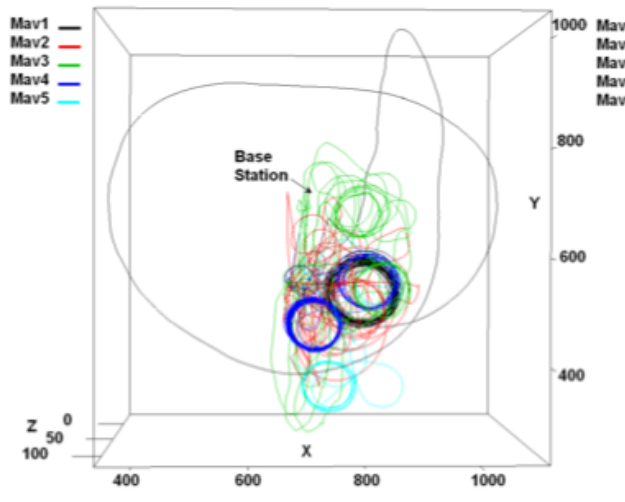

(a) Top view of 5 MAVs loiter circling

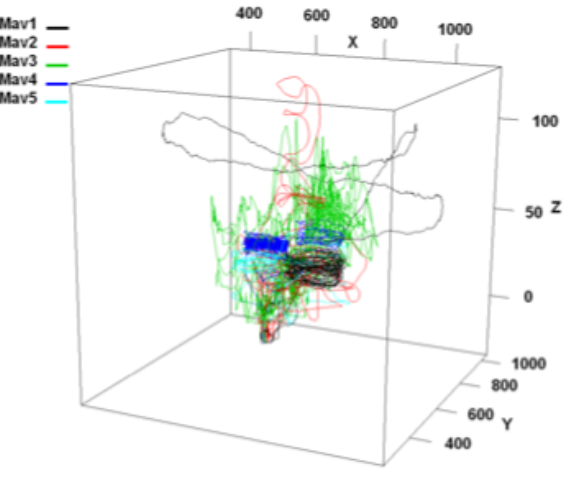

(b) 3D view of 5 MAVs loiter circling

Figure 17.15. Colour-coded trajectories of a five-MAV describing loiter circles around different waypoints (distances given in meters). Image courtesy of Kamran Mohseni, Mobile Sensor Networking Platforms Laboratory, University of Colorado, Boulder.

With these solutions however, robots might not be allocated to the areas where they are most needed. In some cases, it makes more sense to have robots adapt their behaviour based on information gathered during a mission. For example, operators on the ground can monitor the progress of a group of robots in the air and subsequently redirect the robots to areas that need to be investigated. Examples 
using real robots have been shown outdoors using a blimp and two helicopters (Gancet et al., 2005; Merino et al., 2006). However, human driven missions are limited to scenarios with few robots. For example, the operation of a single US Army Hunter or Shadow UAV currently requires the attention of two full time operators while more advanced command and control interfaces have an estimated capacity of less than 5 MAVs per operator (Cummings et al., 2007). An example of an advanced control interface can be seen in Fig. 17.16..

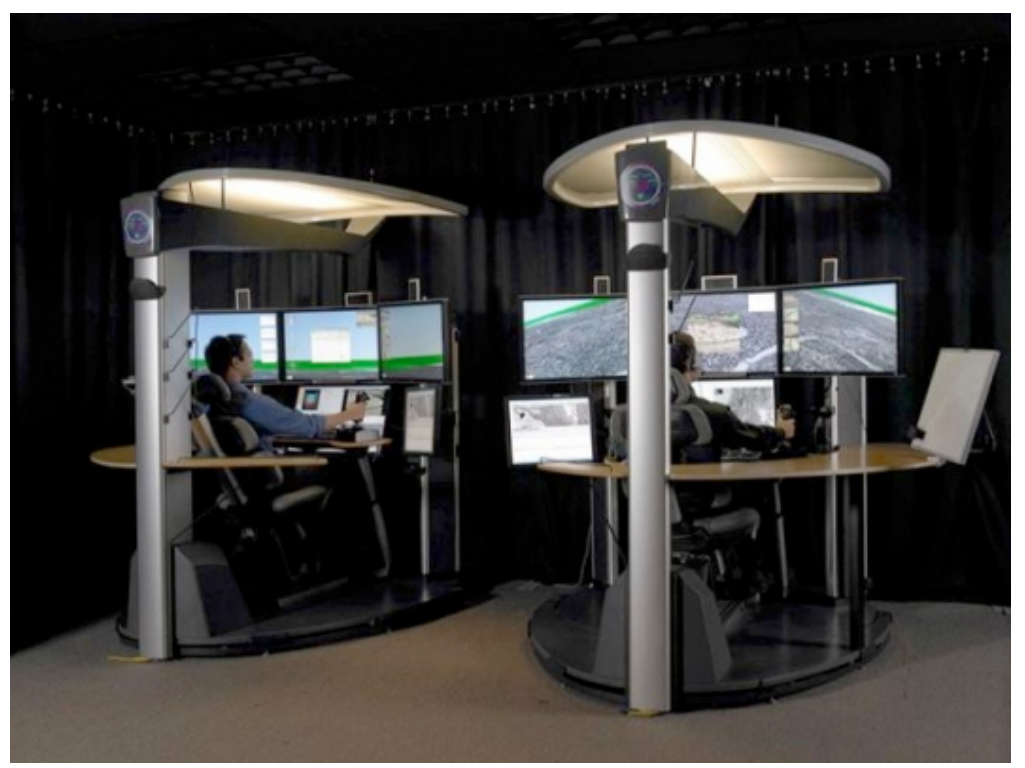

Figure 17.16. Raytheon's Universal Control System for the operation of multiple UAVs (www.raytheon.com, accessed on 01/11/2010).

To increase the amount of robots in the air and therefore their added value to a mission, roboticists are concentrating on giving decision power to the individual robots rather than an operator on the ground. One such strategy commonly used by flying robots is to populate maps that can be used to keep a history of events (position, sensory data, etc.) concerning their neighbours and themselves. In work by Yang et al. (2005), UAVs search for targets in an unknown and uncertain environment based on sensor measurements and positions of all robots in the swarm (evidence). Using this data, robots build evidential maps using probabilistic methods and then use these maps to plan their trajectories so as to maximise their chances of finding the target. In another method loosely inspired from ants, Parunak et al. (2005) and Sauter et al. (2005) have robots search for targets by coordinating their actions through a map containing virtual chemicals (pheromone). Attractive and repulsive pheromones can be deposited on a map or withdrawn from it by a robot and its neighbours as shown in Fig. 17.17.. Furthermore, pheromone evaporates over time meaning that it gradually disappears from the map. Also it can diffuse to nearby areas on the map thereby creating a pheromone gradient. Robots follow attractive pheromone gradients to areas of interest in the environment while depositing repulsive pheromone to prevent robots from monitoring 
overlapping areas (Kuiper and Nadjm-Tehrani, 2006).

Quite similarly to pheromone maps, Peng et al. (2008) have been using UAVs to search an environment by using hormone maps where the attractive and repulsive pheromones are replaced by activator and inhibitor hormones. Finally, Lawrence et al. (2004) create information energy potentials that capture the quality of the measurements made by other robots, their energy loss due to motion and the quality of the ad-hoc network they form. The resulting gradients are then navigated by the robots to map toxic plumes while maintaining a coherent communication network to a central processing unit on the ground. In Uzol et al. (2008), robots also use virtual gradients to track a target while avoiding collisions with buildings and other robots.

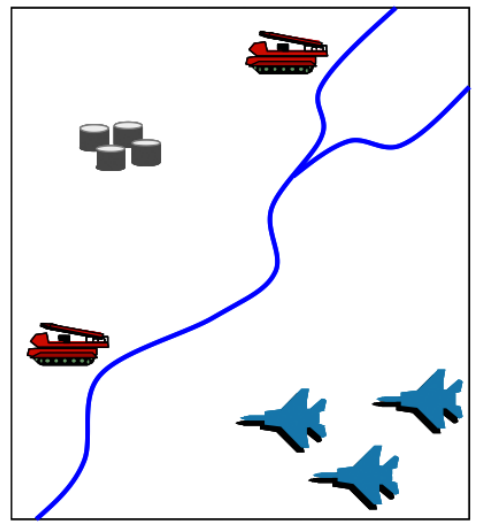

Physical Assets

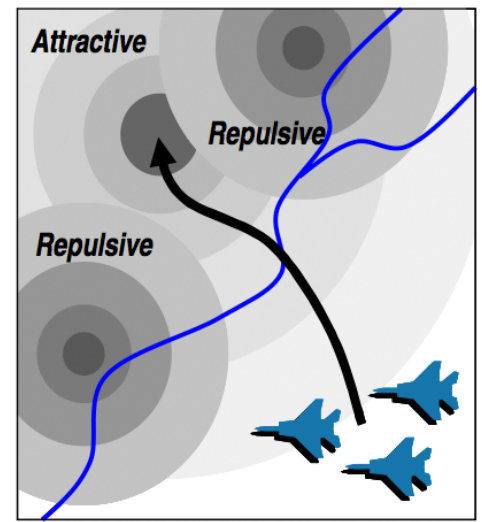

Field Representation

Figure 17.17. Repulsive and attractive pheromone gradients stored on a virtual map used by a swarm of UAVs to find a target while avoiding enemies. Reprinted from (Parunak et al., 2005) with permission of Dr. H. Van Dyke Parunak.

While map-based planning is useful to build a shared view of the advancement of a mission, it requires each robot to know its global position which is not always obvious as explained in Sec. 17.3. For indoor aerial robotic operation or in other GPS-denied environments, researchers have been considering using multiple flying robots for simultaneous localisation and mapping (SLAM). For example, Bryson and Sukkarieh $(2007,2009)$ developed strategies for cooperative SLAM on outdoor UAVs for tasks such as surveillance. A centralised and a decentralised cooperative control system are proposed that actively plan the UAV trajectories in order to maximise the information in the localisation and mapping estimates to build the most accurate map, called active SLAM. The decentralised architecture was slower than the centralised but the final map uncertainty was equal. An advantage of cooperative SLAM is the ability to share the memory and processing requirements between robots, diminishing the computational requirements in decentralised systems. Furthermore collective operation facilitates a super-linear speed-up over single vehicle SLAM (Binns et al., 2002). Multi-robot SLAM however is subject to several challenges that have just started to be ad- 
dressed. Firstly, flying robots often have limited communication thus reducing the amount of map information that can be exchanged between the robots (Bryson and Sukkarieh, 2009; Nettleton et al., 2003). Secondly, SLAM approaches show poor scalability as the operating environment size and complexity increases and as the desired fidelity and resolution of the environment representation increases, requiring greater computational resources (Bailey and Durrant-Whyte, 2006; DurrantWhyte and Bailey, 2006). Finally merging robot maps can be computationally expensive in the case where nothing is known about the relative positions of the robots (Özkucur and Akin, 2010). Sensing relative positioning however is challenging as described in Sec. 17.3.2.

As a solution, recent work has looked at how robots themselves can replace maps by serving as a substrate on which information can be deposited and read from using local communication. For example, in the work by Stirling et al. (2010) shown in Fig. 17.18., inspiration is taken from robotic wireless sensor networks to develop an efficient swarm search algorithm for unknown indoor environments. In this work, robots operate in one of two control states: "Beacons" and "Explorers". Beacons are static robots passively attached to the ceiling to conserve energy and form a robotic sensor network for extended surveillance and monitoring (Roberts et al., 2008). Explorers are actively flying, deploying into the environment being guided by the Beacons. Beacons sense their local environment and communicate with neighbouring Beacons to derive a local communication signal to guide nearby Explorers.
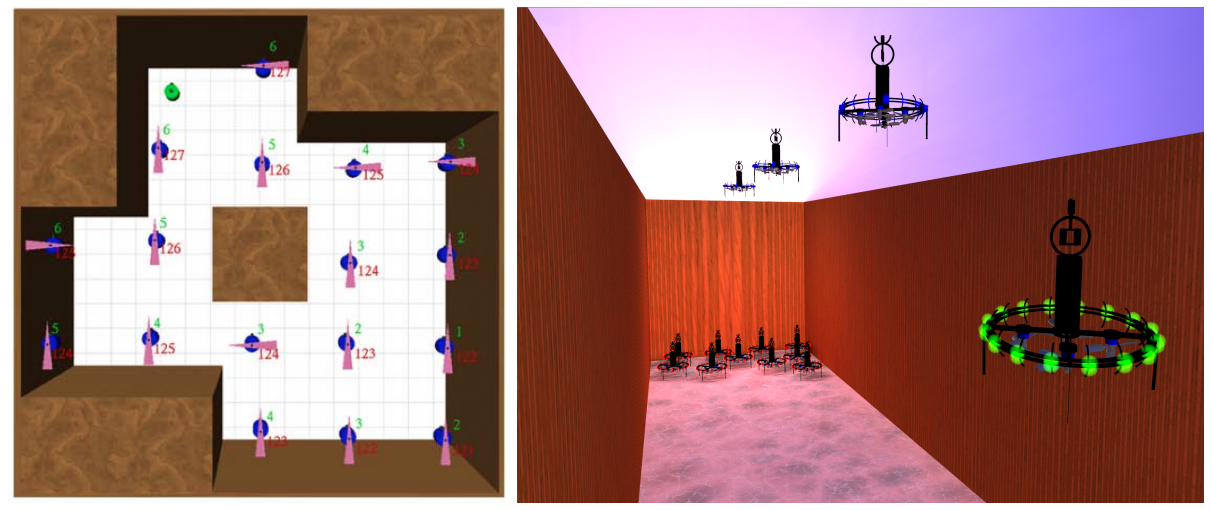

Figure 17.18. Simulation showing Beacons attached to a ceiling signalling directions to an Explorer in green.

In another example, authors take inspiration from ants foraging for food to create controllers for simulated flying robots that must search an area for users and create an ad-hoc network between them (Hauert et al., 2008). Like in nature, robots decide on where to go based on the amount of pheromone present in their environment. To achieve this, robots are separated into two categories, namely "nodeMAVs" that form a grid and serve as a substrate for virtual pheromone and "antMAVs" that can navigate through this grid by reading the pheromone information on the node-MAVs while depositing pheromone on them using local communica- 
tion (Fig. 17.19.). Using this pheromone-based system, robots create dynamic grid structures that reach out from a base from which the robots are launched in search for a user without the need for global or relative positioning.

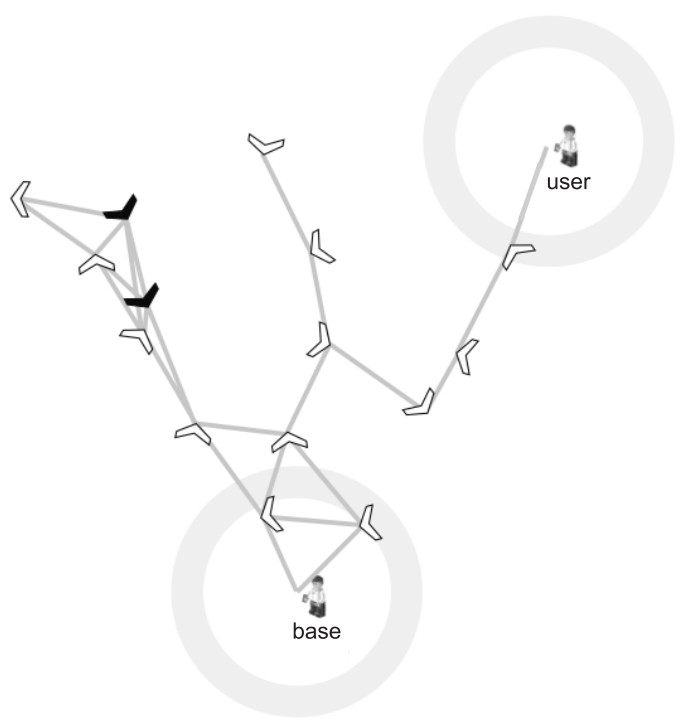

Figure 17.19. Simulator screenshot showing the successful ant-based deployment of flying robots forming an ad-hoc network between a base station and a user. Node-MAVs are white with black borders, ant-MAVs are in solid black, lines represent local communication links and circles around the base and user represent the communication range with noise.

For exploration behaviours to be successful, robots will need to be able to avoid collisions among themselves. This issue however is seldom taken into account during trajectory planning. For this reason, researchers have been concentrating on having robots within collision range negotiate new trajectories in 2D or 3D space (Leven et al., 2010; Šišlák et al., 2007, 2008).

\subsubsection{Flocking}

Flocking allows several robots to move as one without the burden of deploying a single large robot. As an advantage, the failure of any one robot does not cause the entire mission to fail (robustness) and the number of robots can be increased to extend the capability of the system (scalability). Flocking can be used to distribute communication, sensing or computational payloads across different robots (Holland et al., 2005) or to generate visually pleasing aerial 3D patterns (Nembrini et al., 2005). In flocks, the movement of the robots have a direct impact on the movement of other robots. This often leads to interesting effects, such as the avoidance of collisions amongst robots.

Air shows often display the most memorable forms of flocking with army jets performing synchronised ballets above the crowd. Robots have lately been attempting similar manoeuvres in lines (Altshuler et al., 2005; Paul et al., 2008; Vincent and Rubin, 2004; Xue and Zeng, 2009), triangles (Anderson et al., 2008; Paul 
et al., 2008; Xue and Zeng, 2009; Yuan et al., 2007) or diamonds (Xue and Zeng, 2009). To maintain a fixed shape, robots typically need to maintain a predefined distance between themselves and the other robots in the formation. This can be done by pre-programming the trajectory of each robot so that they fly at a predetermined distance from one another (Yuan et al., 2007) in an open-loop fashion or by reactively adjusting the robot's position with respect to its neighbour's (Altshuler et al., 2005; Anderson et al., 2008; Paul et al., 2008; Vincent and Rubin, 2004; Xue and Zeng, 2009).

Aerial flocking can also be observed in nature in birds and insects. Rules driving bird-like flocking (Fig. 17.20.) were proposed by Carl Reynolds (1987) and can be summarised as follows:

- Alignment: robots align their velocity to the average velocity of neighbouring robots.

- Cohesion: robots steer towards the average position of neighbouring robots.

- Separation: robots steer away from neighbouring robots.

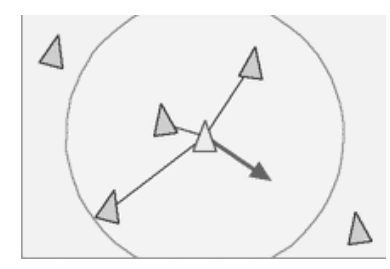

Separation: steer to avoid crowding local flockmates

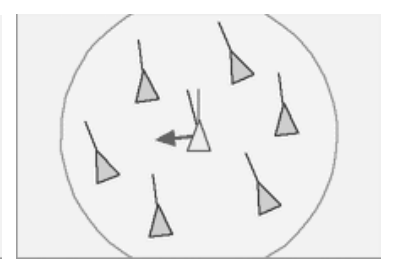

Alignment: steer towards the average heading of local flockmates

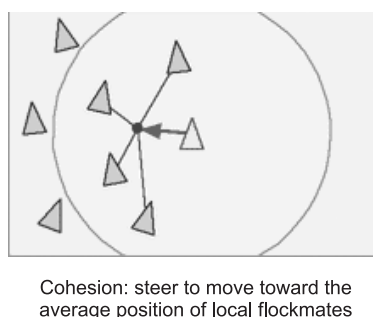

cresion stition

Figure 17.20. Rules for Reynolds' Flocking. The sensing range is shown by a circle (www.red3d.com/cwr/boids/, accessed on 01/11/2010).

Each rule contributes to the final 3D velocity of the robot. The emergent equilibrium in the system allows the robots to avoid collisions or large separations, while having the group advance in a common direction. Several researchers have found that only implementing "separation" and either "alignment" or "cohesion" is often sufficient to achieve coherent flocking (De Nardi, 2004; Park et al., 2003; Sharma and Ghose, 2009). Moreover, work by Kadrovach and Lamont (2001) showed that applying "separation" and "cohesion" to robots with no default forward speed (rotor-crafts) could lead to the formation of a stable grid which could be used to cover an area and serve as a sensor network in the sky. Additional rules can be added to extend the functionalities of Reynolds' flocking. In particular, Basu et al. (2004) looked at how to use a flock of UAVs as an ad-hoc network in the sky that could adapt to its users on the ground. Rules applied include "cohesion" and "separation" as well as a rule to attract robots to good positions above the ground users and a "random walk" rule to repair breaches in the network. Crowther et. al (2004) implemented rules from Reynolds flocking and added two additional ones. The "migration" rule is used to push robots towards a common waypoint and to control the overall swarm. An additional "evasion" rule avoids imminent collisions 
when the separation rule does not suffice. However, only few demonstrations of aerial flocking in reality have been shown with one noticeable example by Welsby and Melhuish (2001) which showed flocking behaviours using four blimps in an indoor environment (Fig. 17.1.).

Similar to the "cohesion" and "separation" rules used for Reynolds' flocking, the field of physicomimetics uses virtual forces inspired from physics to drive robots towards stable formations (Spears et al., 2005). For example, a sensor network made of a hexagonal lattice of MAVs can be formed if each robot reacts to a gravitational force $F=G m_{i} m_{j} / r^{p}$ where $F$ is the magnitude of the force between two neighbouring robots $i$ and $j$ with mass $m, r$ is the distance between them and $p$ and $G$ are user-defined. Given a desired separation $R$ between the robots, the force $F$ is repulsive if $r<R$ and attractive if $r>R$. Spears et al. also designed forces to create square lattices or rules based on the thermodynamics of gases to control a swarm of MAVs moving together within a bounded area while avoiding obstacles for surveillance or coverage applications. Work by Zou et al. (2009) also uses forces to make robots flock while following a desired global trajectory. In work by Chang et al. (2003) flying robots are subject to gyroscopic and braking forces induced by neighbouring robots, thereby creating a collision free environment. For the same purpose, magnetic repulsion laws are used by Sigurd and How (2003) to avoid collisions.

Rather than grids, work by Hauert et al. (2009a) evolved controllers for a swarm of robots that could form chains where each robot's heading is synchronised with its neighbouring robots'. The chain can move in the environment while staying connected to the base from which the robots are launched until a user equipped with an emitter is found. The base and user are then able to communicate via the robot chain forming an ad-hoc network. The swarm behaviour was achieved with robots that know only if they are communicating with the base or user and their own heading.

Flocking has also been exploited in space applications with satellites that need to position themselves precisely and optimally to monitor the Earth and its surrounding atmosphere or for deep space imaging (Kristiansen and Nicklasson, 2009; Truszkowski et al., 2009). Several flocking behaviours have been investigated including cluster-formations where satellites form a dense cloud of robots that can fuse their measurements. Applications include interferometric observations, for creating high-resolution maps of Earth or for finding distant stars and planets. In trailing formations, a leader satellite guides nearby followers equipped with different measurement devices for high-resolution imaging. This formation is valuable to retrieve several sources of measurement of a single event. Finally, constellation formations such as those created by GPS satellites are typically characterised by highly dispersed nodes that provide some service coverage for positioning or communication (Leitner, 2009). Robots in such systems must tightly control their relative distances, velocities and orientations with respect to neighbouring robots. Depending on the application, this tight control can be achieved by physically docking satellites, thereby building a larger satellite. Such modular satellites have been envisioned for a large range of applications from building large 
telescope mirrors or solar panels in space to the build-up of entire space stations (Leitner, 2009). Finally, examples of docking and flocking were shown in reality on-board the International Space Station with three micro-satellites in the scope of the SPHERES project (Nolet et al., 2006).
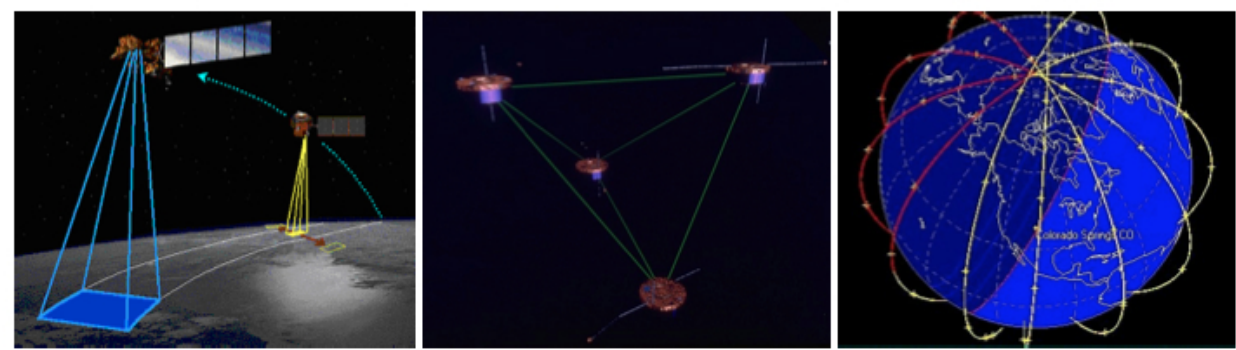

Figure 17.21. The three types of satellite formations: (a) trailing formation, (b) cluster and (c) constellation formation. Image courtesy of Juxi Leitner, Swiss AI Lab, IDSIA, Lugano.

The physical docking of flying robots has also been demonstrated in reality in the Distributed Flight Array project that aims at building aerial modular robots for the study of dynamical systems (Oung et al., 2010). Each module has wheels to drive on the ground and a single propeller. Modules were shown to assemble on the ground, take-off and fly, and then fall back to the ground where the process can resume.

Beyond the practical applications, art has also found its use of flocking through the Mascarillion project which aims towards making a ballet of flying cubes that can change formation in response to the actions of a human artist on the ground (Nembrini et al., 2005). Finally, the Flyfire ${ }^{4}$ project aims at deploying flocks of selforganised helicopters equipped with coloured LEDs as smart pixels to display 3D aerial images.

\subsubsection{Approaches to Controller Design}

Designing controllers for collective aerial systems is challenging because of the lack of established methodologies in this field. Several techniques have already been described in this book.

The most straight forward way to design controllers for collective aerial systems is to adapt classical controllers designed for single robot deployments to multiple robots. Using this approach, one or several operators or pilots become responsible for several flying robots (Gancet et al., 2005; Merino et al., 2006; Murphy et al., 2008). Likewise, waypoint navigation for single robot deployments is applied to several robots with each robot assigned to different areas of interest (Alidaee $e t$ al., 2009; Beard et al., 2006; Bertuccelli et al., 2004; Kovacina et al., 2002; Richards et al., 2002). Classical SLAM algorithms are adapted to merge maps from multiple robots (Bryson and Sukkarieh, 2009, 2007). Search behaviours designed for a single robot (e.g. deterministically search a certain area or follow an information gradient), are

${ }^{4}$ http:/ / senseable.mit.edu/flyfire/ 
conducted by multiple robots to achieve better collective performance (Uzol et al., 2008). Such adapted robot controllers can be driven by a centralised planer or distributed with global or local information as an input.

However, collective aerial robotic applications can benefit from entirely new robot controllers that exploit the local and reactive interactions that may arise between the robots using decentralised controllers. Such controllers are not intended for single robot deployments since the intelligence of the individual controllers emerges from the overall behaviour of the group (Beni, 2005; Sahin, 2005). Because there is often no obvious relation between the local interactions amongst the robots and the desired global behaviour of the group, controllers have typically been designed by experts through trial-and-error (Liu et al., 2007). When possible, roboticists have been turning to nature for inspiration in designing controllers. This makes sense since collective systems have been extensively studied by biologists and physicists. In particular, controllers for flying robots have taken inspiration from pheromone communication in ants (Hauert et al., 2008; Kuiper and Nadjm-Tehrani, 2006; Sauter et al., 2005), flocking by birds (Basu et al., 2004; Crowther, 2004; De Nardi, 2004; Holland et al., 2005; Kadrovach and Lamont, 2001; Reynolds, 1987; Welsby and Melhuish, 2001) and physics (Spears et al., 2005; Zou et al., 2009).

More recently, researchers have been using artificial evolution to automatically design controllers for flying robots (Gaudiano et al., 2005; Hauert et al., 2009a; Richards et al., 2005; Ruini and Cangelosi, 2009). This strategy has the potential to automatically find unthought of controllers that are simple and efficient. However, artificial evolution takes time and evolved controllers are only suitable for the environment in which they were evolved. To overcome these shortcomings, it has been proposed to reverse-engineer evolved controllers. Reverse-engineering consists in systematically analysing how an evolved controller works and then capturing its main functionalities in a hand-designed controller. Resulting controllers have the advantage of being easier to understand than evolved controllers and can even be mathematically modelled and therefore easily parameterised for a variety of scenarios (Hauert et al., 2009b). In recent work, Hauert et al. used reverse-engineering to design controllers for area-coverage (Hauert et al., 2009b), communication relay (Hauert et al., 2009b) and for the synchronisation and steering of groups of flying robots (Hauert et al., 2010a). An outdoor demonstration of 5 swinglets (Fig. 17.4.) synchronizing their heading while being steered is shown in Fig. 17.22..

\subsection{Conclusion}

This chapter provides a very broad overview of existing techniques allowing to design functional aerial collective systems, starting with the flying platforms and going up to the design of swarm controllers. Although many researchers have been pioneering this field over the past decade, very little demonstrations have been conducted with physical flying platforms. A few noticeable exceptions are Allred et al. (2007); Beard et al. (2006); Gancet et al. (2005); Hauert et al. (2010a); Hoffmann et al. (2004); How et al. (2004); Leven et al. (2010); Melhuish and Welsby (2002). 

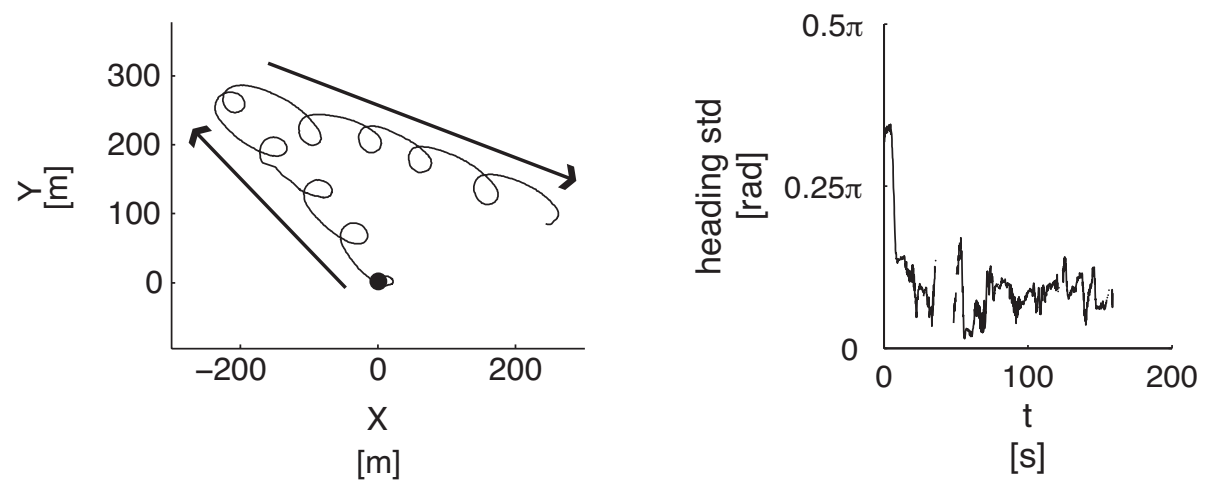

Figure 17.22. Demonstration of heading synchronisation and steering of five real flying robots in an outdoor experiment. Left: mean trajectory of the robots. Right: standard deviation on the robot headings. Two phases are shown here, in phase I, the robot group is directed to the West. In phase II, robots are directed to turn around and proceed East. Notice that the robots' headings remain synchronized throughout the experiment and that the trajectories of the 5 robots are very similar, which is why it is possible to plot such a mean trajectory.

However, none of these projects have demonstrated swarms of more than five robots. This matter of fact indicates that there are still a few challenges ahead. The first one is certainly the availability of lightweight, safe and affordable flying platforms featuring good manoeuvrability, crash-robustness and even self-recovery capabilities in case of crash or mid-air collisions. The currently available energy storage methods also present a strong limitation. Energy sharing and harvesting techniques should be further developed in the near future in order to achieve long-lasting experiments that would be closer to commercially-viable applications. Relative positioning and communication techniques are also not yet up to the task, especially when it comes to small and lightweight flying systems. Therefore, enabling coordination among flying robots is still very challenging and as such often takes a large part of the research and development resources. Once these challenges will be solved and commercially available platforms will become available, some of the numerous methods for flying robot coordination that have been proposed so far and tested in simulation will finally find their way to the sky. This will naturally pave the way towards achieving economically viable aerial collective systems.

\section{Acknowledgements}

This work has been supported by the following projects and funding agencies: The Future Emerging Technologies (FET IST-022888) “Swarmanoid Project" funded by the European Commission; the Swiss National Science Foundation (grant 200020116149); the Swiss Innovation Promotion Agency CTI (project no. 11116.1 PFNMNM); Armasuisse, competence sector Science + Technology for the Swiss Federal Department of Defence, Civil Protection and Sports; and lastly the FET programme within the Seventh Framework Programme for Research of the European Commission, under the CURVACE project (FET-Open grant number: 237940). The authors 
17.5. Conclusion $\mid \mathbf{3 7}$

would like to thank all those that gave assistance in writing this chapter. 
38 Aerial Collective Systems 


\section{Bibliography}

Achtelik, M., Bachrach, A., He, R., Prentice, S. and Roy, N. (2009). Autonomous navigation and exploration of a quadrotor helicopter in GPS-denied indoor environments, in First Symposium on Indoor Flight Issues (AUVSI, Arlington), pp. 1-12.

Ahrens, S., Levine, D., Andrews, G. and How, J. P. (2009). Vision-based guidance and control of a hovering vehicle in unknown, GPS-denied environments, in Proceedings of the IEEE International Conference on Robotics and Automation (IEEE Press), pp. 3155-3160.

Alidaee, B., Wang, H. and Landram, F. (2009). A Note on Integer Programming Formulations of the Real-Time Optimal Scheduling and Flight Path Selection of UAVs, IEEE Transactions on Control Systems Technology 17, 4, pp. 839-843.

Allen, M. and Lin, V. (2007). Guidance and control of an autonomous soaring vehicle with flight test results, in Proceedings of the 45th AIAA Aerospace Sciences Meeting and Exhibit, pp. 1-26, AIAA paper AIAA-2007-867.

Allred, J., Hasan, A. B., Panichsakul, S., Pisano, W., Gray, P., Huang, J., Han, R., Lawrence, D. and Mohseni, K. (2007). SensorFlock: an airborne wireless sensor network of micro-air vehicles, in Proceedings of the 5th International Conference on Embedded Networked Sensor Systems (ACM Press), pp. 117-129.

Altshuler, Y., Yanovsky, V., Wagner, I. and Bruckstein, A. (2008). Efficient cooperative search of smart targets using UAV swarms, Robotica 26, 4, pp. 551-557.

Altshuler, Y., Yanovsky, V., Wagner, I. A. and Bruckstein, A. (2005). The cooperative hunters - efficient cooperative search for smart targets using UAV swarms, in Proceedings of the Second International Conference on Informatics in Control, Automation and Robotics, pp. 165-170.

Ambrosino, G., Ariola, M., Ciniglio, U., Corraro, F., Pironti, A. and Virgilio, M. (2006). Algorithms for 3D UAV path generation and tracking, in Proceedings of the IEEE Conference on Decision and Control (CDC) (IEEE Press), pp. 5275-5280.

Anderson, B., Fidan, B., Yu, C. and Walle, D. (2008). UAV formation control: Theory and application, in Recent Advances in Learning and Control, Lecture Notes in Control and Information Sciences, Vol. 371 (Springer Berlin), pp. 15-33.

Andrievsky, B. R. and Fradkov, A. L. (2003). Combined adaptive controller for UAV guidance, in Proceedings of the European Control Conference (ECC), pp. 71-79.

Artieda, J., Sebastián, J. M., Campoy, P., Correa, J. F., Mondragón, I. F., Martínez, C. and Olivares, M. (2009). Visual 3-D SLAM from UAVs, Journal of Intelligent and Robotic Systems 55, 4-5, pp. 299-321.

Bachmann, R. J., Boria, F. J., Ifju, P., Quinn, R., Kline, J. E. and Vaidyanathan, R. (2005). Utility of a sensor platform capable of aerial and terrestrial locomotion, in Proceedings of the IEEE/ASME International Conference on Advanced Intelligent Mechatronics (IEEE Press), pp. 1581-1586.

Bailey, T. and Durrant-Whyte, H. (2006). Simultaneous localization and mapping (SLAM): part II, IEEE Robotics $\mathcal{E}$ Automation Magazine 13, 3, pp. 108-117.

Balanis, C. A. (1997). Fundamental Parameters of Antennas, Vol. Second Edition (John Wiley \& Sons), pp. 28-53.

Basu, P., Redi, J. and Shurbanov, V. (2004). Coordinated flocking of UAVs for improved connectivity of mobile ground nodes, in Proceedings of the IEEE Military Communications Conference, Vol. 3 (IEEE Press), pp. 1628-1634.

Beard, R., Kingston, D., Quigley, M., Snyder, D., Christiansen, R. and Johnson, W. (2005). 
Autonomous vehicle technologies for small fixed-wing UAVs, Journal of Aerospace Computing, Information, and Communication 2, 1, pp. 92-108.

Beard, R. W., Mclain, T. W., Nelson, D. B., Kingston, D. and Johanson, D. (2006). Decentralized cooperative aerial surveillance using fixed-wing miniature UAVs, Proceedings of the IEEE 94, 7, pp. 1306-1324.

Beni, G. (2005). From swarm intelligence to swarm robotics, in Swarm Robotics, Lecture Notes in Computer Science, Vol. 3342 (Springer Berlin), pp. 1-9.

Bermudez i Badia, S., Pyk, P. and Verschure, P. (2005). A biologically based flight control system for a blimp-based UAV, in Proceedings of the IEEE International Conference on Robotics and Automation (IEEE Press), pp. 3053-3059.

Berne, M. and Pogorel, G. (2003). Challenges for Wi-Fi, business models and spectrum issues, Annals of Telecommunications 58, 3-4, pp. 576-583.

Bertuccelli, L., Alighanbari, M. and How, J. (2004). Robust planning for coupled cooperative UAV missions, in Proceedings of the IEEE Conference on Decision and Control, Vol. 17 (IEEE Press), pp. 2917-2922.

Beyeler, A., Zufferey, J.-C. and Floreano, D. (2009). Vision-based control of near-obstacle flight, Autonomous Robots 27, 3, pp. 201-219.

Binns, L. A., Valachis, D., Anderson, S., Gough, D. W., Nicholson, D. and Greenway, P. (2002). Distributed SLAM, in Signal Processing, Sensor Fusion, and Target Recognition XI, SPIE proceedings series, Vol. 4729 (SPIE), pp. 62-68.

Bouabdallah, S., Murrieri, P. and Siegwart, R. (2005). Towards autonomous indoor micro VTOL, Autonomous Robots 18, 2, pp. 171-183.

Bouabdallah, S. and Siegwart, R. (2007). Advances in Unmanned Aerial Vehicles, chap. Design and Control of a Miniature Quadrotor (Springer Press), pp. 171-210.

Brisset, P., Drouin, A., Gorraz, M., Huard, P.-S. and Tyler, J. (2006). The Paparazzi Solution, in Proceedings of the $2^{\text {nd }}$ Micro Air Vehicle Conference and Competition (EMAV'06) (Braunschweig, Germany).

Brisset, P. and Hattenberger, G. (2008). Multi-UAV control with the paparazzi system, in Proceedings of the first conference on Humans Operating Unmanned Systems.

Bryson, M. and Sukkarieh, S. (2007). Co-operative localisation and mapping for multiple UAVs in unknown environments, in Proceedings of the Aerospace Conference (IEEE Press), pp. 1-12.

Bryson, M. and Sukkarieh, S. (2009). Architectures for cooperative airborne simultaneous localisation and mapping, Journal of Intelligent and Robotics Systems 55, 4-5, pp. 267297.

Byrne, J., Cosgrove, M. and Mehra, R. (2006). Stereo based obstacle detection for an unmanned air vehicle, in Proceedings of the IEEE International Conference on Robotics and Automation (ICRA) (IEEE Press), pp. 2830-2835.

Carnie, R., Walker, R. and Corke, P. (2006). Image processing algorithms for UAV "sense and avoid", in Proceedings of the IEEE International Conference on Robotics and Automation (IEEE Press), pp. 2848-2853.

Chang, D., Shadden, S., Marsden, J. and Olfati-Saber, R. (2003). Collision avoidance for multiple agent systems, in Proceedings of the 42nd IEEE Conference on Decision and Control (IEEE Press), pp. 539-543.

Cheng, C.-M., Hsiao, P.-H., Kung, H. T. and Vlah, D. (2006). Performance measurement of 802.11a wireless links from UAV to ground nodes with various antenna orientations, in Proceedings of the 15th IEEE International Conference on Computer Communications and Networks (IEEE Press), pp. 303-308.

Chichka, D. and Speyer, J. (1998). Solar-powered, formation-enhanced aerial vehicle systems for sustained endurance, in Proceedings of the 1998 American Control Conference, Vol. 2 (IEEE Press), pp. 684-688.

Cole, D., Goktogan, A. and Sukkarieh, S. (2006). The demonstration of a cooperative control architecture for UAV teams, Experimental Robotics 39, pp. 501-510.

Cory, R. and Tedrake, R. (2008). Experiments in fixed-wing UAV perching, in Proceedings 
of the AIAA Conference on Guidance, Navigation, and Control, AIAA paper AIAA-20087256.

Crowther, B. (2004). Rule-based guidance for flight vehicle flocking, Proceedings of the Institute of Mechanical Engineers, Part G: Journal of Aerospace Engineering 218, 2, pp. 111-124.

Cummings, M., Nehme, C., Crandall, J. and Mitchell, P. (2007). Predicting operator capacity for supervisory control of multiple uavs, in Innovations in Intelligent Machines - 1, Studies in Computational Intelligence, Vol. 70 (Springer Berlin), pp. 11-37.

De Nardi, R. (2004). Flocking of UAVs Software model and limited vision simulations, Ph.D. thesis, University of Padua Italy.

De Nardi, R., Holland, O., Woods, J. and Clark, A. (2006). SwarMAV: A swarm of miniature aerial vehicles, in Proceedings of the 21st International UAV Systems Conference.

DeLima, P., York, G. and Pack, D. (2006). Localization of ground targets using a flying sensor network, in Proceedings of the IEEE International Conference on Sensor Networks, Ubiquitous, and Trustworthy Computing-Vol 1 (IEEE Press), pp. 194-199.

Desbiens, A. L., Asbeck, A. T. and Cutkosky, M. R. (2009). Scansorial landing and perching, in Proceedings of the 14th International Symposium on Robotics Research, pp. 1-14.

Dixon, C., Frew, E. and Argrow, B. (2005). Radio leashing of an unmanned aircraft, in Proceedings of Infotech@Aerospace, AIAA paper AIAA-2005-7030.

Dixon, C. and Frew, E. W. (2009). Maintaining optimal communication chains in robotic sensor networks using mobility control, Mobile Networks and Applications Journal 14, 3, pp. 281-291.

Ducard, G. and D'Andrea, R. (2009). Autonomous quadrotor flight using a vision system and accommodating frames misalignment, in Proceedings of the IEEE International Symposium on Industrial Embedded Systems (IEEE Press), pp. 261-264.

Dudek, G. and Jenkin, M. (2008). Inertial sensors, GPS, and odometry, in Springer Handbook of Robotics (Springer Berlin Heidelberg), pp. 477-490.

Durrant-Whyte, H. and Bailey, T. (2006). Simultaneous localization and mapping (SLAM): part I, IEEE Robotics \& Automation Magazine 13, 2, pp. 99-110.

Etkin, B. and Reid, L. D. (1995). Dynamics of Flight: Stability and Control, 3rd edn. (Wiley).

Euston, M., Coote, P., Mahony, R., Kim, J. and Hamel, T. (2008). A complementary filter for attitude estimation of a fixed-wing UAV, in Proceedings of the IEEE/RSJ International Conference on Intelligent Robots and Systems (IEEE Press), pp. 340-345.

Feron, E. and Johnson, E. N. (2008). Aerial Robotics (Springer), pp. 1009-1029.

Fielding, C. and Luckner, R. (2000). Industrial considerations for flight control, in Flight Control Systems, Vol. 57, 1st edn., chap. 1 (The Institution of Electrical Engineers (IEE)), p. 28.

Floreano, D., Zufferey, J.-C., Srinivasan, M. V. and Ellington, C. P. (2009). Flying Insects and Robots (Springer).

Frew, E., Lawrence, D. A., Dixon, C., Elston, J. and Pisano, W. J. (2007). Lyapunov guidance vector fields for unmanned aircraft applications, in Proceedings of the American Control Conference (ACC), pp. 371-376.

Gancet, J., Hattenberger, G., Alami, R. and Lacroix, S. (2005). Task planning and control for a multi-UAV system: architecture and algorithms, in Proceedings of the IEEE International Conference on Intelligent Robots and Systems (IEEE Press), pp. 1017-1022.

Gaudiano, P., Bonabeau, E. and Shargel, B. (2005). Evolving behaviors for a swarm of unmanned air vehicles, in Proceedings of the IEEE Swarm Intelligence Symposium (IEEE Press), pp. 317-324.

Gordon, J. (2006). Principles of helicopter aerodynamics (Cambridge University Press).

Green, W. E. and Oh, P. Y. (2005). A MAV that flies like an airplane and hovers like a helicopter, in Proceedings of the IEEE/ASME International Conference on Advanced Intelligent Mechatronics (IEEE Press), pp. 699-704.

Green, W. E. and Oh, P. Y. (2006). Autonomous hovering of a fixed-wing micro air vehicle, in Proceedings of the IEEE International Conference on Robotics and Automation (IEEE Press), pp. 2164-2169. 
Green, W. E. and Oh, P. Y. (2008). Optic flow based collision avoidance on a hybrid MAV, IEEE Robotics and Automation Magazine 15, 1, pp. 96-103.

Griffiths, S., Saunders, J., Curtis, A., Barber, B., McLain, T. and Beard, R. (2006). Maximizing miniature aerial vehicles, IEEE Robotics and Automation Magazine 13, 3, pp. 34-43.

Griffiths, S., Saunders, J., Curtis, A., McLain, T. and Beard, R. (2007). Obstacle and terrain avoidance for miniature aerial vehicles, in K. Valavanis (ed.), Advances in Unmanned Aerial Vehicles: State of the Art and the Road to Autonomy, Vol. 33, chap. 7 (Springer), pp. 213-244.

Grzonka, S., Grisetti, G. and Burgard, W. (2009). Towards a navigation system for autonomous indoor flying, in Proceedings of the International Conference on Robotics and Automation (IEEE Press), pp. 2878-2883.

Guglieri, G., Quagliotti, F. B. and Speciale, G. (2008). Optimal trajectory tracking for an autonomous UAV, Automatic Control in Aerospace (online journal) 1, pp. 1-9.

Gurdan, D., Stumpf, J., Achtelik, M., Doth, K., Hirzinger, G. and Rus, D. (2007). Energyefficient autonomous four-rotor flying robot controlled at $1 \mathrm{khz}$, in Proceedings of the 2007 IEEE International Conference on Robotics and Automation (IEEE Press), pp. 361366.

Hauert, S., Leven, S., Zufferey, J.-C. and Floreano, D. (2010a). Beat-based synchronization and steering for groups of fixed-wing flying robots, in Proceedings of the 10th International Symposium on Distributed Autonomous Robotics Systems.

Hauert, S., Leven, S., Zufferey, J.-C. and Floreano, D. (2010b). Communication-based leashing of real flying robots, in Proceedings of the IEEE International Conference on Robotics and Automation (IEEE Press).

Hauert, S., Winkler, L., Zufferey, J.-C. and Floreano, D. (2008). Ant-based swarming with positionless micro air vehicles for communication relay, Swarm Intelligence 2, 2-4, pp. 167-188.

Hauert, S., Zufferey, J.-C. and Floreano, D. (2009a). Evolved swarming without positioning information: an application in aerial communication relay, Autonomous Robots 26, 1, pp. 21-32.

Hauert, S., Zufferey, J.-C. and Floreano, D. (2009b). Reverse-engineering of artificially evolved controllers for swarms of robots, in Proceedings of the IEEE Congress on Evolutionary Computation (IEEE Press), pp. 55-61.

Hirosue, W., Ookura, A. and Sunada, S. (2003). A study of a coaxial helicopter(ii): Analysis on effects of a stabilizer bar on fuselage motion, Proceedings of the 41st Aircraft Symposium of the Japan Society for Aeronautical and Space Sciences 41, 1, pp. 283-286.

Hoffmann, G., Huang, H., Waslander, S. L. and Tomlin, C. J. (2007). Quadrotor helicopter flight dynamics and control: Theory and experiment, in Proceedings of the AIAA Guidance, Navigation, and Control Conference, pp. 2007-6461.

Hoffmann, G., Rajnarayan, D., Waslander, S., Dostal, D., Jang, J. and Tomlin, C. (2004). The Stanford testbed of autonomous rotorcraft for multi agent control (STARMAC), in Proceedings of the 23rd Digital Avionics Systems Conference, Vol. 2 (IEEE Press), pp. 1-10.

Hoffmann, G., Waslander, S. and Tomlin, C. (2008). Quadrotor helicopter trajectory tracking control, in Proceedings of the AIAA Guidance, Navigation, and Control Conference, pp. $1-14$.

Holland, O., Woods, J., De Nardi, R. and Clark, A. (2005). Beyond swarm intelligence: the UltraSwarm, in Proceedings of the IEEE Swarm Intelligence Symposium (IEEE Press), pp. 217-224.

How, J., Bethke, B., Frank, A., Dale, D. and Vian, J. (2008). Real-time indoor autonomous vehicle test environment, IEEE Control Systems Magazine 28, 2, pp. 51-64.

How, J., King, E. and Kuwata, Y. (2004). Flight demonstrations of cooperative control for UAV teams, in Proceedings of the AIAA 3rd Unmanned Unlimited Technical Conference, Workshop and Exhibit, September, AIAA paper AIAA-2004-6490.

Howard, A., Mataric, M. J. and Sukhatme, G. S. (2002). Mobile sensor network deployment using potential fields: A distributed, scalable solution to the area coverage problem, 
Distributed Autonomous Robotic Systems 5, pp. 299-308.

Iida, F. (2003). Biologically inspired visual odometer for navigation of a flying robot, Robotics and Autonomous Systems 44, pp. 201-208.

Jang, J. S. and Tomlin, C. G. (2001). Autopilot Design for the Stanford DragonFly UAV: Validation through Hardware-in-the-Loop Simulation, in Proeedings of the AIAA Guidance, Navigation and Control Conference (GNC) (Montreal), AIAA paper AIAA-20014179.

Kadrovach, B. A. and Lamont, G. B. (2001). Design and analysis of swarm-based sensor systems, in Proceedings of the IEEE Midwest Symposium on Circuits and Systems, Vol. 1 (IEEE Press), pp. 487-490.

Kendoul, F., Zhenyu, Y. and Nonami, K. (2009). Embedded autopilot for accurate waypoint navigation and trajectory tracking: Application to miniature rotorcraft UAVs, in Proceedings of the IEEE International Conference on Robotics and Automation (IEEE Press), pp. 2332-2338.

Kim, J.-H., Wishart, S. and Sukkarieh, S. (2006). Real-time navigation, guidance, and control of a uav using low-cost sensors, in Field and Service Robotics, Springer Tracts in Advanced Robotics, Vol. 24 (Springer Berlin), pp. 299-309.

Kim, S., Knoll, T. and Scholz, O. (2007). Feasibility of inductive communication between millimeter-sized wireless robots, IEEE Transactions on Robotics 23, 3, pp. 605-609.

Kingston, D. B. and Beard, R. W. (2004). Real-time attitude and position estimation for small uavs using low-cost sensors, in Proceedings of the AIAA $3^{\text {rd }}$ Unmanned Unlimited Systems Conference and Workshop, AIAA paper AIAA-2004-6533.

Klaptocz, A., Boutinard Rouelle, G., Briod, A., Zufferey, J.-C. and Floreano, D. (2010). An indoor flying platform with collision robustness and self-recovery, in Proceedings of the IEEE/RSJ International Conference on Robotics and Automation (IEEE Press), pp. 33493354 .

Knoebel, N. B., Osborne, S. R., Matthews, J. S., Eldredge, A. M. and Beard, R. W. (2006). Computationally simple model reference adaptive control for miniature air vehicles, in Proceedings of the American Control Conference, pp. 5978-5983.

Kovac, M., Germann, J. M., Huerzeler, C., Siegwart, R. and Floreano, D. (2010). A perching mechanism for micro aerial vehicles, Journal of Micro-Nano Mechatronics 5, 3-4, pp. 77-91.

Kovacina, M., Palmer, D., Yang, G. and Vaidyanathan, R. (2002). Multi-agent control algorithms for chemical cloud detection and mapping using unmanned air vehicles, in Proceedings of the IEEE/RSJ International Conference on Intelligent Robots and System, Vol. 3 (IEEE Press), pp. 2782-2788.

Kristiansen, R. and Nicklasson, P. J. (2009). Spacecraft formation flying: A review and new results on state feedback control, Acta Astronautica 65, 11-12, pp. 1537-1552.

Kubo, M. and Melhuish, C. (2004). Robot trophallaxis: Managing energy autonomy in multiple robots, in Proceedings of Towards Autonomous Robotic Systems, pp. 77-84.

Kuiper, E. and Nadjm-Tehrani, S. (2006). Mobility models for UAV group reconnaissance applications, in Proceedings of the IEEE International Conference on Wireless and Mobile Communications (IEEE Press), p. 33.

Kundu, A., Jang, J. H., Gil, J. H., Jung, C. R., Lee, H. R., Kim, S. H., Ku, B. and Oh, Y. S. (2007). Micro-fuel cells-current development and applications, Journal of Power Sources 170, 1, pp. 67-78.

Lanzisera, S., Lin, D. T. and Pister, K. S. J. (2006). RF time of flight ranging for wireless sensor network localization, in Proceedings of the 4th Workshop on Intelligent Solutions in Embedded Systems, pp. 1-12.

Lawrence, D., Donahue, R., Mohseni, K. and Han, R. (2004). Information energy for sensorreactive UAV flock control, in Proceedings of the AIAA "Unmanned Unlimited" Technical Conference, AIAA paper AIAA-2004-6530.

Lawrence, D. A., Frew, E. W. and Pisano, W. J. (2008). Lyapunov vector fields for autonomous unmanned aircraft flight control, Journal of Guidance, Control and Dynamics 31, 5, pp. 1220-1229. 
Leitner, J. (2009). Multi-robot cooperation in space: A survey, Advanced Technologies for Enhanced Quality of Life, pp. 144-151.

Lentink, D. (2008). Exploring the Biofluiddynamics of Swimming and Flight, Ph.D. thesis, Experimental Zoology Group, Wageningen University.

Lentink, D., Jongerius, S. and Bradshaw, N. (2009). The scalable design of flapping microair vehicles inspired by insect flight, in D. Floreano, J.-C. Zufferey, M. Srinivasan and C. Ellington (eds.), Flying Insects and Robots, chap. 14 (Springer), pp. 185-205.

Leven, S., Zufferey, J.-C. and Floreano, D. (2009). A Minimalist Control Strategy for Small UAVs, in Proceedings of the IEEE/RSJ International Conference on Intelligent Robots and Systems (IEEE Press), pp. 2873-2878.

Leven, S., Zufferey, J.-C. and Floreano, D. (2010). Mid-air collision avoidance in dense collective aeiral systems, Journal of Field Robotics In press.

Levine, W. S. (1996). Sample-Rate Selection, Vol. Series II, chap. 16 (CRC \& IEEE Press), pp. 313-321.

Liu, W., Winfield, A. F. T. and Sa, J. (2007). Modelling swarm robotic systems: A case study in collective foraging, in Proceedings of Towards Autonomous Robotic Systems, pp. 25-32.

Lizarraga, M. I., Ilstrup, D. M., Elkaim, G. H. and Davis, J. (2008). Aerial photography using a nokia N95, in Proceedings of the World Congress on Engineering and Computer Science.

Lo, Y. T. and Lee, S. W. (1993). Antenna theory, in Antenna Handbook (Kluwer Academic Publishers).

Lupashin, S., Schoellig, A., Sherback, M. and D'Andrea, R. (2010). A simple learning strategy for high-speed quadrocopter multi-flips, in Proceedings of the International Conference on Robotics and Automation, pp. 1642-1648.

Marins, J. L., Yun, X., Bachmann, E. R., McGhee, R. B. and Zyda, M. J. (2001). An extended kalman filter for quaternion-based orientation estimation using MARG sensors, in Proceedings of the IEEE/RSJ International Conference on Intelligent Robots and Systems (IEEE Press), pp. 2003-2011.

Matsue, A., Hirosue, W., Tokutake, A., Sundada, S. and Ohkura, A. (2005). Navigation of small and lightweight helicopter, Transactions of the Japan Society for Aeronautical and Space Sciences 48 , pp. 177-179.

McLurkin, J. and Smith, J. (2007). Distributed algorithms for dispersion in indoor environments using a swarm of autonomous mobile robots, in R. Alami, R. Chatila and H. Asama (eds.), Distributed Autonomous Robotic Systems 6 (Springer Japan), pp. 399408.

Mei, Y., Lu, Y.-H., Hu, Y. C. and Lee, C. S. G. (2004). Energy-efficient motion planning for mobile robots, in Proceedings of the International Conference on Robotics and Automation, Vol. 5 (IEEE Press), pp. 4344-4349.

Mei, Y., Lu, Y.-H., Hu, Y. C. and Lee, C. S. G. (2005). A case study of mobile robot's energy consumption and conservation techniques, in Proceedings of the 12th International Conference on Advanced Robotics (IEEE Press), pp. 492-497.

Melhuish, C. and Welsby, J. (2002). Gradient ascent with a group of minimalist real robots: Implementing secondary swarming, in Proceedings of the IEEE International Conference on Systems, Man and Cybernetics, Vol. 2 (IEEE Press), pp. 509-514.

Merino, L., Caballero, F., Martínez-de Dios, J. R., Ferruz, J. and Ollero, A. (2006). A cooperative perception system for multiple UAVs: application to automatic detection of forest fires, Journal of Field Robotics 23, pp. 165-184.

Meyer, J.-A. and Filliat, D. (2003). Map-based navigation in mobile robots: II. a review of map-learning and path-planning strategies, Cognitive Systems Research 4, 4, pp. 283317.

Montesano, L., Montano, L. and Burgard, W. (2004). Relative localization for pairs of robots based on unidentifiable moving features, in Proceedings of the IEEE/RSJ International Conference on Intelligent Robots and Systems, Vol. 2 (IEEE Press), pp. 1537-1543.

Murphy, R. R., Pratt, K. S. and Burke, J. L. (2008). Crew roles and operational protocols for rotary-wing micro-UAVs in close urban environments, in Proceedings of the 3rd 
ACM/IEEE International Conference on Human Robot Interaction (ACM Press, New York, USA), pp. 73-80.

Nakamura, T., Oohara, M., Ogasawara, T. and Ishiguro, H. (2003). Fast self-localization method for mobile robots using multiple omnidirectional vision sensors, Machine Vision and Applications 14, 2, pp. 129-138.

Nelson, D., Barber, D., McLain, T. and Beard, R. (2007). Vector field path following for miniature air vehicles, IEEE Transactions on Robotics 23, 3, pp. 519-529.

Nembrini, J., Reeves, N., Poncet, E., Martinoli, A. and Winfield, A. F. T. (2005). Mascarillon: Flying swarm intelligence for architectural research, in Proceedings of the IEEE Swarm Intelligence Symposium (IEEE Press), pp. 225-232.

Nettleton, E., Thrun, S. and Durrant-Whyte, H. (2003). Decentralised SLAM with lowbandwidth communication for teams of airborne vehicles, in Proceedings of the International Conference on Field and Service Robotics.

Nolet, S., Saenz-Otero, A., Miller, D. and Fejzic, A. (2006). SPHERES operations aboard the ISS: Maturation of GN\&C algorithms in microgravity, in Proceedings of the AAS Guidance and Control Conference, aAS 07042.

Noth, A., Engel, W. and Siegwart, R. (2006a). Design of an ultra-lightweight autonomous solar airplane for continuous flight, in P. Corke and S. Sukkariah (eds.), Field and Service Robotics, Springer Tracts in Advanced Robotics, Vol. 25 (Springer Berlin), pp. 441-452.

Noth, A., Engel, W. and Siegwart, R. (2006b). Flying solo and solar to mars, IEEE Robotics and Automation Magazine 13, 3, pp. 44-52.

Oh, P. Y., Green, W. E. and Barrows, G. (2004). Neural nets and optic flow for autonomous micro-air-vehicle navigation, in Proceedings of the ASME International Mechanical Engineering Congress and Exposition, pp. 1279-1285.

Oh, P. Y., Joyce, M. and Gallagher, J. (2005). Designing an aerial robot for hover-and-stare surveillance, in Proceedings of the IEEE International Conference on Advanced Robotics (IEEE Press), pp. 303-308.

O'Hara, K. J., Nathuji, R., Raj, H., Schwan, K. and Balch, T. (2006). Autopower: toward energy-aware software systems for distributed mobile robots, in Proceedings of the IEEE International Conference on Robotics and Automation (IEEE Press), pp. 2757-2762.

Ohkura, A., Tokutake, A. and Sundada, S. (2005). Autonomous hovering of a small helicopter, Transactions of the Japan Society for Aeronautical and Space Sciences 53, pp. 376378.

Oung, R., Bourgault, F., Donovan, M. and D'Andrea, R. (2010). The Distributed Flight Array, in Proceedings of the IEEE Conference on Robotics and Automation (IEEE Press), pp. 601607.

Oyekan, J. and Huosheng, H. (2009). Toward bacterial swarm for environmental monitoring, in Proceedings of the IEEE International Conference on Automation and Logistics, August (IEEE Press), pp. 399-404.

Özkucur, N. E. and Akin, H. L. (2010). Cooperative multi-robot map merging using fastSLAM, RoboCup 2009: Robot Soccer World Cup XIII 5949, pp. 449-460.

Park, C.-S., Tahk, M. and Bang, H. (2003). Multiple aerial vehicle formation using swarm intelligence, in Proceedings of the AIAA Guidance, Navigation, and Control Conference and Exhibit, AIAA paper AIAA-2003-5729.

Parunak, H. V. D., Brueckner, S. A. and Sauter, J. (2005). Digital pheromones for coordination of unmanned vehicles, in D. Weyns, H. V. D. Parunak and F. Michel (eds.), Environments for Multi-Agent Systems, Lecture Notes in Computer Science, Vol. 3374 (Springer Berlin / Heidelberg), pp. 246-263.

Paul, T., Krogstad, T. and Gravdahl, J. (2008). Modelling of UAV formation flight using 3D potential field, Simulation Modelling Practice and Theory 16, 9, pp. 1453-1462.

Payton, D., Estkowski, R. and Howard, M. (2004). Pheromone robotics and the logic of virtual pheromones, in E. Şahin and W. Spears (eds.), Swarm Robotics, Lecture Notes in Computer Science, Vol. 3342 (Springer Berlin), pp. 45-57.

Peng, H., Li, Y., Wang, L. and Shen, L. (2008). Hormone-inspired cooperative control for 
multiple UAVs wide area search, in Proceedings of the 4th International Conference on Intelligent Computing: Advanced Intelligent Computing Theories and Applications-with Aspects of Theoretical and Methodological Issues, Lecture Notes in Computer Science, Vol. 5226 (Springer Berlin), pp. 808-816.

Pisano, W. J. and Lawrence, D. A. (2007). Autonomous UAV control using a 3-sensor autopilot, in Proceedings of the AIAA Infotech@Aerospace Conference and Exhibit, AIAA paper AIAA-2007-2756.

Pratt, R. W. (2000). Flight Control Systems, Vol. 57, 1st edn. (The Institution of Electrical Engineers (IEE)).

Pugh, J., Raemy, X., Favre, C., Falconi, R. and Martinoli, A. (2009). A fast on-board relative positioning module for multi-robot systems, IEEE Transactions on Mechatronics, Focused Section on Mechatronics in Multi-Robot Systems 14, 2, pp. 151-162.

Qu, Z. (2009). Cooperative Control of Dynamical Systems: Applications to Autonomous Vehicles (Springer).

Reynolds, C. W. (1987). Flocks, herds and schools: a distributed behavioral model, in Proceedings of the 14th Annual Conference on Computer Graphics and Interactive Techniques, Vol. 21 (ACM Press), pp. 25-34.

Richards, A., Bellingham, J., Tillerson, M. and How, J. (2002). Co-ordination and control of multiple UAVs, in Proceedings of the AIAA Guidance, Navigation and Control Conference, AIAA Paper AIAA-2002-4588.

Richards, M. D., Whitley, D. and Beveridge, J. R. (2005). Evolving cooperative strategies for UAV teams, in Proceedings of the Genetic and Evolutionary Computation Conference, Vol. 2 (ACM Press), pp. 1721-1728.

Rivard, F., Bisson, J., Michaud, F. and Letourneau, D. (2008). Ultrasonic relative positioning for multi-robot systems, in Proceedings of the IEEE International Conference on Robotics and Automation (IEEE Press), pp. 323-328.

Roberts, J. F., Stirling, T. S., Zufferey, J.-C. and Floreano, D. (2009). 2.5D infrared range and bearing system for collective robotics, in Proceedings of the International Conference on Intelligent Robots and Systems (IEEE Press), pp. 3659-3664.

Roberts, J. F., Zufferey, J.-C. and Floreano, D. (2008). Energy management for indoor hovering robots, in Proceedings of the International Conference on Intelligent Robots and Systems (IEEE Press), pp. 1242-1247.

Romero, H., Salazar, S., Sanchez, A. and Lozano, R. (2007). A new UAV configuration having eight rotors: Dynamical model and real-time control, in Proceedings of the 46th IEEE Conference on Decision and Control (IEEE Press), pp. 6418-6423.

Rosenfeld, A., Kaminka, G. A. and Kraus, S. (2006). A study of scalability properties in robotic teams, in P. Scerri, R. Vincent and R. Mailler (eds.), Coordination of Large-Scale Multiagent Systems (Springer US), pp. 27-51.

Ruini, F. and Cangelosi, A. (2009). Extending the evolutionary robotics approach to flying machines: An application to MAV teams, Neural Networks 22, 5-6, pp. 812-821.

Rybski, P., Larson, A., Lindahl, M. and Gini, M. (1998). Performance evaluation of multiple robots in a search and retrieval task, in Proceedings of the Workshop on Artificial Intelligence and Manufacturing (AAAI Press, Menlo Park), pp. 153-160.

Sahin, E. (2005). Swarm robotics: From sources of inspiration to domains of application, in Swarm Robotics, Lecture Notes in Computer Science, Vol. 3342 (Springer Berlin), pp. $10-20$.

Salazar, S., Romero, H., Lozano, R. and Castillo, P. (2009). Modeling and real-time stabilization of an aircraft having eight rotors, Journal of Intelligent and Robotic Systems 57, pp. 455-470.

Sauter, J. A., Matthews, R., Parunak, H. V. D. and Brueckner, S. A. (2005). Performance of digital pheromones for swarming vehicle control, in Proceedings of the International Joint Conference on Autonomous Agents and Multi-Agent Systems (ACM Press), pp. 903910.

Scherer, S., Singh, S., Chamberlain, L. and Elgersma, M. (2008). Flying fast and low among 
obstacles: Methodology and experiments, The International Journal of Robotics Research 27, 5, pp. 549-574.

Seybold, J. S. (2005). Chapter 7: Near Earth Propagation Models, Vol. 1 (John Wiley \& Sons), pp. 134-146.

Shakernia, O., Chen, W.-Z. and Raska, V. M. (2005). Passive ranging for UAV sense and avoid applications, in Proceedings of Infotech@Aerospace, AIAA paper AIAA-2005-7179.

Sharma, R. K. and Ghose, D. (2009). Collision avoidance between UAV clusters using swarm intelligence, International Journal of Systems Science 40, 5, pp. 521-538.

Shen, J., Moh, S. and Chung, I. (2008). Routing protocols in delay tolerant networks: A comparative survey, in Proceedings of the 23rd International Technical Conference on Circuits/Systems, Computers and Communications, pp. 1577-1580.

Shore, D. and Bodson, M. (2004). Flight testing of a reconfigurable control system on an unmanned aircraft, in Proceedings of the American Control Conference, Vol. 6, pp. 37473752.

Shoval, S. and Borenstein, J. (2001). Measuring the relative position and orientation between two mobile robot with binaural sonar, in Proceedings of the ANS 9th International Topical Meeting on Robotics and Remote Systems, pp. 1-12.

Siegwart, R. and Nourbakhsh, I. R. (2004). Introduction to Autonomous Mobile Robots (MIT Press, Cambridge).

Sigurd, K. and How, J. (2003). UAV trajectory design using total field collision avoidance, in Proceedings of the AIAA Guidance, Navigation and Control Conference, AIAA paper AIAA-2003-5728.

Spears, W. M., Spears, D. F., Heil, R., Kerr, W. and Hettiarachchi, S. (2005). An overview of physicomimetics, in Swarm Robotics, Lecture Notes in Computer Science, Vol. 3342 (Springer, Berlin), pp. 84-97.

Spletzer, J., Das, A., Fierro, R., Taylor, C., Kumar, V. and Ostrowski, J. (2001). Cooperative localization and control for multi-robot manipulation, in Proceedings of the IEEE/RSJ International Conference on Intelligent Robots and Systems (IEEE Press), pp. 631-636.

Steder, B., Grisetti, G., Stachniss, C. and Burgard, W. (2008). Visual SLAM for flying vehicles, IEEE Transactions on Robotics 24, 5, pp. 1088-1093.

Stevens, B. L. and Lewis, F. L. (2003). Aircraft Control and Simulation, 2nd edn. (Wiley).

Stirling, T. and Floreano, D. (2010a). Energy Efficient Swarm Deployment for Search in Unknown Environments, in M. Dorigo (ed.), Proceedings of the 7th International Conference on Swarm Intelligence (ANTS 2010), Lecture Notes in Computer Science (LNCS) (Springer-Verlag, Berlin), pp. 562-563.

Stirling, T. and Floreano, D. (2010b). Energy-time efficiency in aerial swarm deployment, in Proceedings of the 10th International Symposium on Distributed Autonomous Robotics Systems.

Stirling, T., Wischmann, S. and Floreano, D. (2010). Energy-efficient indoor search by swarms of simulated flying robots without global information, Swarm Intelligence 4, 2, pp. 117-143.

Tennekes, H. (1997). The simple science of flight: from insects to jumbo jets (The MIT Press).

Thrun, S., Montemerlo, M., Dahlkamp, H., Strohband, S., Dupont, C., Bradski, G., Davies, B. and Mahoney, P. (2006). Stanley: The robot that won the darpa grand challenge, Field Robotics 23, 9, pp. 661-692.

Thrun, S., Montemerlo, M., Koller, D., Wegbreit, B., Nieto, J. and Nebot, E. (2004). FastSLAM: An efficient solution to the simultaneous localization and mapping problem with unknown data association, Journal of Machine Learning Research 4, 3, pp. 380-407.

Timcenko, V., Stojanovic, M. and Rakas, S. B. (2009). Manet routing protocols vs. mobility models: performance analysis and comparison, Proceedings of the 9th WSEAS international conference on Applied informatics and communications, pp. 271-276.

Törnqvist, D., Schön, T., Karlsson, R. and Gustafsson, F. (2009). Particle filter SLAM with high dimensional vehicle model, Journal of Intelligent and Robotic Systems 55, 4, pp. 249-266. 
Tozer, T., Grace, D., Thompson, J. and Baynham, P. (2000). UAVs and HAPs-potential convergence for military communications, IEE Seminar Digests 2000, 24, pp. 10/1-10/6.

Truszkowski, W., Hallock, H., Rouff, C., Karlin, J., Rash, J., Hinchey, M. and Sterritt, R. (2009). Swarms in space missions, in Autonomous and Autonomic Systems: With Applications to NASA Intelligent Spacecraft Operations and Exploration Systems, NASA Monographs in Systems and Software Engineering (Springer), pp. 207-221.

Utt, J., McCalmont, J. and Deschenes, M. (2005). Development of a sense and avoid system, in Proceedings of Infotech@Aerospace, AIAA paper AIAA-2005-7177.

Uzol, O., Yavrucuk, I. and Sezer-Uzol, N. (2008). Collaborative target tracking for swarming MAVs using potential fields and panel methods, in Proceedings of the AIAA Guidance, Navigation and Control Conference, AIAA paper AIAA-2008-7167.

Valenti, M., Bethke, B., How, J.-P., Farias, D.-P. and Vian, J. (2007). Embedding health management into mission tasking for UAV teams, in Proceedings of the American Control Conference (IEEE Press), pp. 5777-5783.

Van Der Zwaan, S., Bernardino, A. and Santos-Victor, J. (2002). Visual station keeping for floating robots in unstructured environments, Robotics and Autonomous Systems 39, 34, pp. 145-155.

Vincent, P. and Rubin, I. (2004). A framework and analysis for cooperative search using UAV swarms, in Proceedings of the ACM Symposium on Applied Computing (ACM Press), pp. 79-86.

Viquerat, A., Blackhall, L., Reid, A. and Sukkarieh, S. (2007). Reactive collision avoidance for unmanned aerial vehicles using doppler radar, in C. Laugier and R. Siegwart (eds.), Proceedings of the 6th Conference on Field and Service Robotics, Springer Tracts in Advanced Robotics, Vol. 42 (Springer Berlin), pp. 245-254.

Šišlák, D., Samek, J. and Pěchouček, M. (2008). Decentralized algorithms for collision avoidance in airspace, in Proceedings of the 7th International Joint Conference on Autonomous Agents and Multiagent Systems, pp. 543-550.

Šišlák, D., Volf, P., Komenda, A., Samek, J. and Pěchouček, M. (2007). Agent-based multilayer collision avoidance to unmanned aerial vehicles, in Proceedings of the International Conference on Integration of Knowledge Intensive Multi-Agent Systems (IEEE Press), pp. 365-370.

Wang, T., Wang, B., Wei, H., Cao, Y., Wang, M. and Shao, Z. (2008). Staying-alive and energyefficient path planning for mobile robots, in Proceedings of the American Control Conference (IEEE Press), pp. 868-873.

Waslander, S. L., Hoffmann, G., Jang, J. S. and Tomlin, C. J. (2005). Multi-agent X4-flyer testbed design: Integral sliding mode vs. reinforcement learning, in Proceedings of the IEEE International Conference on Intelligent Robots and Systems (IEEE Press).

Welsby, J. and Melhuish, C. (2001). Autonomous minimalist following in three dimensions: A study with small-scale dirigibles, in Proceedings of Towards Intelligent Mobile Robots. Technical.

Wibowo, S. B., Klepal, M. and Pesch, D. (2009). Time of flight ranging using off-the-self IEEE802.11 WiFi tags, in Proceedings of the International Conference on Positioning and Context-Awareness.

Wickenheiser, A. and Garcia, E. (2008). Optimization of perching maneuvers through vehicle morphing, Journal of Guidance Control and Dynamics 31, 4, pp. 815-823.

$\mathrm{Wu}, \mathrm{H} .$, Sun, D., Zhu, H. and Zhou, Z. (2005). An autonomous flight control strategy study of a small-sized unmanned air vehicle, IEICE Transactions on Electronics E88-C, 10.

Wzorek, M., Landén, D. and Doherty, P. (2006). GSM technology as a communication media for an autonomous unmanned aerial vehicle, in Proceedings of the 21st Bristol UAV Systems Conference.

Xue, Z. and Zeng, J. (2009). Formation control numerical simulations of geometric patterns for unmanned autonomous vehicles with swarm dynamical methodologies, in Proceedings of the International Conference on Measuring Technology and Mechatronics Automation (IEEE Press), pp. 477-482. 
Yang, Y., Minai, A. A. and Polycarpou, M. M. (2005). Evidential map-building approaches for multi-UAV cooperative search, in Proceedings of the IEEE American Control Conference (IEEE Press), pp. 116-121.

York, G. W. and Pack, D. J. (2008). Cooperative persistent surveillance search algorithms using multiple unmanned aerial vehicles, in D. Grundel, R. Murphey, P. Pardalos and O. Prokopyev (eds.), Cooperative Networks: Control and Optimization (Edward Elgar Publishing, Cheltenham), pp. 279-290.

Yuan, H., Gottesman, V., Falash, M., Qu, Z., Pollak, E. and Chunyu, J. (2007). Cooperative formation flying in autonomous unmanned air systems with application to training, in P. Pardalos, R. Murphey, D. Grundel and M. Hirsch (eds.), Advances in Cooperative Control and Optimization, Lecture Notes in Control and Information Sciences, Vol. 369 (Springer Berlin), pp. 203-219.

Zarzhitsky, D. and Spears, D. (2005). Swarm approach to chemical source localization, in Proceedings of the IEEE International Conference on Systems, Man and Cybernetics (IEEE Press), p. 14351440.

Zeimpekis, V., Giaglis, G. M. and Lekakos, G. (2003). A taxonomy of indoor and outdoor positioning techniques for mobile location services, ACM SIGecom Exchange 3, 4, pp. $19-27$.

Zhang, H. and Ostrowski, J. (1998). Visual servoing with dynamics: Control of an unmanned blimp, in Proceedings of the IEEE International Conference on Robotics and Automation (IEEE Press), pp. 618-623.

Zou, Y., Pagilla, P. and Ratliff, R. (2009). Distributed formation flight control using constraint forces, Journal of Guidance, Control, and Dynamics 32, 1, pp. 112-120.

Zufferey, J.-C. (2008). Bio-inspired Flying Robots: Experimental Synthesis of Autonomous Indoor Flyers (EPFL/CRC Press).

Zufferey, J.-C., Beyeler, A. and Floreano, D. (2009). Optic Flow to Steer and Avoid Collisions in $3 D$, chap. 6 (Springer), pp. 73-86.

Zufferey, J.-C., Beyeler, A. and Floreano, D. (2010). Autonomous flight at low altitude using light sensors and little computational power, International Journal of Micro Air Vehicles 2, 2, pp. 107-117.

Zufferey, J.-C., Guanella, A., Beyeler, A. and Floreano, D. (2006a). Flying over the reality gap: From simulated to real indoor airships, Autonomous Robots 21, 3, pp. 243-254.

Zufferey, J.-C., Klaptocz, A., Beyeler, A., Nicoud, J.-D. and Floreano, D. (2006b). A 10-gram microflyer for vision-based indoor navigation, in Proceedings of the IEEE/RSJ International Conference on Intelligent Robots and Systems (IEEE Press), pp. 3267-3272. 